\title{
How do red-eyed treefrog embryos sense motion in predator attacks? Assessing the role of vestibular mechanoreception \\ Running title: Vestibular motion-sensing in ovo
}

\author{
Authors: \\ Panamá \\ *Author for correspondence (jungj@,bu.edu) \\ Contact info for corresponding author: \\ Julie Jung \\ Department of Biology \\ 5 Cummington Mall \\ Boston, MA \\ 02215 \\ (510) 439-7614 \\ jungj@,bu.edu
}

Julie Jung $^{1 *}$, Su J. Kim ${ }^{1}$, Sonia M. Pérez Arias ${ }^{1}$, James G. McDaniel ${ }^{2}$, Karen M. Warkentin ${ }^{1,3}$

${ }^{1}$ Department of Biology, Boston University, 5 Cummington Mall, Boston, MA, 02215, USA

${ }^{2}$ Department of Mechanical Engineering, 110 Cummington Mall, Boston, MA, 02215, USA

${ }^{3}$ Smithsonian Tropical Research Institute, Apartado Postal 0843-03092, Panamá, República de

Key words: environmentally cued hatching, phenotypic plasticity, vestibular system, biotremology, predator risk, ontogeny.

1 Red-eyed treefrogs' hatching responses to predator attacks, vibration playbacks, and egg-jiggling 2 appear when vestibular function develops. Ear development may be a key limiting factor in the 3 onset of mechanosensory-cued hatching. 
ABSTRACT

The widespread ability to alter hatching timing in response to environmental cues can serve as a defense against threats to eggs. Arboreal embryos of red-eyed treefrogs, Agalychnis callidryas, hatch up to $30 \%$ prematurely to escape predation. This escape-hatching response is cued by physical disturbance of eggs during attacks, including vibrations or motion, and thus depends critically on mechanosensory ability. Predator-induced hatching appears later in development than flooding-induced, hypoxia-cued hatching; thus, its onset is not constrained by the development of hatching ability. It may, instead, reflect the development of mechanosensor function. We hypothesize that vestibular mechanoreception mediates escape-hatching in snake attacks, and that the developmental period when hatching-competent embryos fail to flee from snakes reflects a sensory constraint. We assessed the ontogenetic congruence of escape-hatching responses and an indicator of vestibular function, the vestibulo-ocular reflex (VOR), in three ways. First, we measured VOR in two developmental series of embryos 3-7 days old to compare with the published ontogeny of escape success in attacks. Second, during the period of greatest variation in VOR and escape success, we compared hatching responses and VOR across sibships. Finally, in developmental series, we compared the response of individual embryos to a simulated attack cue with their VOR. The onset of VOR and hatching responses were largely concurrent at all three scales. Moreover, latency to hatch in simulated attacks decreased with increasing VOR. These results are consistent with a key role of the vestibular system in the escape-hatching response of $A$. callidryas embryos to attacks.

8 Hatching is an essential embryo behavior that mediates the transition between two distinct stages of life, in the egg and post-hatching environments, when developing animals are exposed to different risks and opportunities. Variation in either environment can affect when is

31 the best time to hatch. Environmentally cued hatching allows embryos to respond adaptively to

32 their local environment by altering the timing of their hatching (Sih and Moore, 1993;

33 Warkentin, 1995). Recent syntheses reveal that cued hatching responses are phylogenetically 34 widespread (Warkentin, 2011a). Physical disturbance of eggs is particularly common as a cue for 
35 hatching, as observed in invertebrates (Endo et al., 2018; Mukai et al., 2014; Nishide and

36 Tanaka, 2016; Oyarzun and Strathmann, 2011; Whittington and Kearn, 1988), fishes (Martin et

37 al., 2011), amphibians (Buckley et al., 2005; Gomez-Mestre et al., 2008; Goyes Vallejos et al.,

38 2018; Touchon et al., 2011; Warkentin, 1995; Warkentin, 2000; Warkentin, 2011b), and reptiles

39 (Doody, 2011; Doody and Paull, 2013; Doody et al., 2012). Physical disturbance cues can

40 function in antipredator responses, conspecific-cued hatching, host-cued hatching of parasites,

41 and embryo responses to physical conditions (Warkentin, 2011a; Warkentin, 2011b). Physical

42 disturbance may be a particularly useful cue to impending predation of terrestrial eggs, since

43 predators cannot eat eggs without touching and moving them, and terrestrial embryos appear to

44 have less opportunity to receive chemical early warning cues than do aquatic embryos.

45 To our knowledge, the mechanosensory system mediating hatching responses to physical

46 disturbance cues has not been assessed for any embryos. Indeed, we know relatively little about

47 the developmental onset of mechanoreception, compared to its mature function, across taxa (Hill,

48 2008). In vertebrates, the predominant motion-detection system is the vestibular system of the

49 inner ear. Otic mechanoreceptors appear during embryonic development in fishes (Becerra and

50 Anadon, 1993; Bever and Fekete, 2002; Haddon and Lewis, 1996), amphibians (Fritzsch, 1996;

51 Quick and Serrano, 2005), chicks (Alsina and Whitfield, 2017; Liang et al., 2010), mice

52 (Fritzsch, 2003; Fritzsch et al., 2002), and humans (Fritzsch et al., 1998). Thus they could

53 potentially mediate mechanosensory-cued hatching. In fishes and amphibians, the lateral line

54 system also develops before hatching (Bever et al., 2003; Hill, 2008; Nieuwkoop and Faber,

55 1956; Stone, 1933; Thomas et al., 2015) and thus might play a role in mediating cued-hatching

56 responses. Understanding the sensors that mediate cue perception is a key part of understanding

57 any cued behavior and may be particularly crucial early in ontogeny, when both sensory abilities

58 and behavior are changing rapidly. Unlike adults, with fully developed sensory systems,

59 embryos' ability to respond to particular cue types is constrained by the need for adequate prior

60 development of the relevant sensors. It is essential to identify these sensors and assess their

61 ontogeny in order to determine when developmental changes in behavior reflect the easing of

62 sensory constraints and to understand the information available to embryos at different

63 developmental stages. Understanding sensory ontogeny will also facilitate inquiry into other

64 sources of developmental changes in behavior, such as ontogenetic adaptation of decision rules

65 (Warkentin et al., 2019). 
Red-eyed treefrog embryos, Agalychnis callidryas (Cope 1862), are tractable study

67 organisms for research on predator-induced, mechanosensory-cued hatching of terrestrial eggs.

68 Females lay gelatinous egg clutches upon leaves and other substrates overhanging ponds, such

69 that hatching tadpoles generally fall into the water below as soon as they hatch (Gomez-Mestre

70 et al., 2008; Pyburn, 1970). Thus hatching, for these embryos, entails a change from arboreal to

71 aquatic habitats with concomitant changes in selection pressures and potential predators

72 (Warkentin, 1995). Predation is the largest cause of mortality for A. callidryas embryos

73 monitored at ponds in Costa Rica and Panama, and attacks during the last third of the typical

74 undisturbed embryonic period induce rapid escape-hatching responses of embryos (Gomez-

75 Mestre and Warkentin, 2007; Warkentin, 1995; Warkentin, 2000). Embryos hatch in response to

76 physical disturbance by predators and, at least for snake attacks, playback of recorded attack-

77 vibrations is sufficient to elicit premature hatching (Hughey et al., 2015; Warkentin, 2005).

78 Embryos can hatch within seconds by rapidly releasing hatching enzymes to digest a small hole

79 in the membrane, then squeezing through it (Cohen et al., 2016). Embryos also hatch

80 prematurely in response to flooding, cued by hypoxia (Warkentin, 2002), and drying, based on

81 unknown cues (Salica et al., 2017).

82 We recently discovered that the developmental onset of hatching responses to hypoxia

83 and mechanosensory cues differs in A. callidryas (Warkentin et al., 2017). Specifically, there is a

84 period of development when embryos are competent to hatch, as demonstrated by their

85 consistent hatching response to strong hypoxia and the presence of hatching gland cells, yet still

86 unresponsive to mechanosensory disturbance cues or natural predators (Cohen et al., 2019;

87 Warkentin et al., 2017). Up to 10\% of eggs laid can be consumed during this period (Gomez-

88 Mestre and Warkentin, 2007; Warkentin, 1995; Warkentin, 2000; Warkentin et al., 2017),

89 suggesting that an earlier onset of escape-hatching responses to predators could be beneficial.

90 The existence of this hatching-competent but unresponsive-to-predators period indicates that

91 something beyond hatching ability limits the onset of the anti-predator response (Warkentin et

92 al., 2017). The survival cost of early hatching decreases gradually, over days, not hours, of

93 development (Warkentin, 1995; Warkentin, 1999; Willink et al., 2014); this suggests that

94 changes in adaptive embryo decisions are unlikely to impose a narrow developmental limit on

95 the onset of the anti-predator response. Instead, the rapid developmental increase in response to a

96 simulated attack cue, from 0-100\% hatching over a few hours (Warkentin et al., 2017), suggests 
97 a sensory constraint may limit embryos ability to detect attacks. Thus, as an initial step to assess

98 what sensory system mediates vibration perception in attacks, we looked for ontogenetic

99 congruence of sensor development and the onset of the escape-hatching behavior.

100 We hypothesize that $A$. callidryas embryos use inner ear mechanoreceptors to sense

101 motion cues. If the vestibular system is the primary mechanism by which red-eyed treefrog

102 embryos sense the physical disturbance of their egg clutches, and is required to perceive predator

103 attacks, its development may limit the onset of escape-hatching responses in this context. To

104 assess this, we looked for developmental correlations between escape hatching behavior and a

105 marker of vestibular function, the vestibulo-ocular reflex (VOR). This reflex generates eye

106 movements that compensate for head movement, producing a more stable image in the retina

107 (Straka, 2010). VOR can be used as a convenient behavioral indicator of vestibular function, as it

108 depends critically on vestibular system development (Jen, 2009). The development of roll-

109 induced VOR has been extensively studied in the frog Xenopus laevis (Horn et al., 1986a; Horn

110 et al., 1986b; Horn, 2006; Horn and Gabriel, 2011; Rayer and Horn, 1986), the fish Oreochromis

111 mossambicus (Sebastian et al., 2001; Sebastian and Horn, 1999), and the salamander Pleurodeles

112 waltl (Gabriel et al., 2012), demonstrating that VOR amplitude is a sensitive indicator of

113 vestibular function. In these aquatic species, modifications of vestibular input either by vestibular

114 lesions (Horn et al., 1986b; Rayer and Horn, 1986; Schaefer and Meyer, 1974) or by altered

115 gravitational conditions during critical periods of vestibular system development (Gabriel et al.,

116 2012; Horn, 2006; Horn and Gabriel, 2011; Sebastian et al., 2001) reliably lead to significant

117 reductions in VOR.

118 We began by quantifying the basic ontogeny of VOR in A. callidryas embryos. We

119 predicted that the developmental onset of VOR (increase in magnitude from absent to

120 consistently strong) would align with the previously documented ontogeny of A. callidryas'

121 escape-hatching success in predator attacks. In attacks by egg-predatory snakes and wasps, we

122 have never observed hatching before about 2.5 days prematurely. In our Panamanian study

123 population, escape-hatching success is zero at age $3 \mathrm{~d}$, present but relatively low and variable at

$1244 \mathrm{~d}$, and consistently high thereafter, with spontaneous hatching in the evening at $6 \mathrm{~d}$ (Almanzar

125 and Warkentin, 2018; Hite et al., 2018; Warkentin, 2000; Warkentin et al., 2006a). In

126 Warkentin's 1990s research on the Osa Peninsula, Costa Rica, with A. callidryas developing

127 more slowly under cooler conditions, predator-induced hatching began at age $5 \mathrm{~d}$, with 
128 spontaneous hatching in the evening at $7 \mathrm{~d}$ (Gomez-Mestre and Warkentin, 2007; Gomez-Mestre

129 et al., 2008; Warkentin, 1995). Accordingly, if a strong VOR were present at the onset of

130 hatching competence at $3 \mathrm{~d}$, vestibular system development would be implausible as a constraint

131 underlying the later onset of attack-induced hatching. Moreover, assuming that VOR is a reliable

132 marker for vestibular function, if VOR onset were clearly later than the onset of escape responses

133 to attacks (e.g., not present until 5 d), it would reject a key role for the vestibular system in

134 sensing attacks.

135 Next, focusing on the developmental period of greatest variation in VOR and hatching

136 response, we directly compared the hatching responses of egg clutches to vibration playback

137 with the VOR of a subset of hatched and unhatched individuals from each clutch. During this

138 period of high variation, and potentially rapid developmental change, we predicted a positive

139 relationship between magnitude of VOR and hatching response, with some threshold value

140 below which vestibular function is insufficient to cue hatching.

141 Finally, we applied a simulated predator attack cue to individual eggs to compare

142 hatching responses of embryos to their VOR, as clutches developed. Here as well, we predicted

143 that the onset of vestibular function would match the onset of hatching responses. A lack of

144 correlation of VOR magnitude and hatching response across either clutches or individuals,

145 during the period of high variation and response onsets, would suggest that these are

146 developmentally independent events that simply happen to occur during the same general period.

147 The presence of correlated VOR and hatching responses both among clutches and among

148 individuals would be consistent with a functional linkage.

\section{METHODS}

152 Egg clutch collection and care

153 We collected 0-3 d old A. callidryas egg clutches and the leaves on which they were laid

154 from the Experimental Pond in Gamboa, Panama (9.120894 N, 79.704015 W). Clutches were

155 brought to a nearby ambient temperature and humidity laboratory at the Smithsonian Tropical

156 Research Institute, mounted on plastic cards for support, positioned over aged tap water in plastic

157 cups, and misted with rainwater frequently to maintain hydration. Tests of hatching responses

158 were conducted in the ambient-conditions laboratory, and individual hatchlings were tested for 
159 VOR in an adjacent air-conditioned laboratory. All embryos used were morphologically normal,

160 in developmental synchrony with siblings in their clutch, and in intact, turgid eggs at the start of

161 the experiment. Most clutches are laid between $10 \mathrm{pm}$ and $2 \mathrm{am}$, so we assign embryos to daily

162 age-classes and report developmental timing starting from midnight of their oviposition night

163 (Warkentin, 2002; Warkentin et al., 2005). Across the onset of hatching competence, tested

164 individuals were staged based on morphological markers described in Warkentin et al. (2017).

165 Some specimens were preserved for morphological studies (to be presented elsewhere) and all

166 other hatchlings were returned to their pond. This research was conducted under permits from

167 the Panamanian Environmental Ministry (SC/A-15-14, SE/A-46-15) and approved by the

168 Institutional Animal Care and Use Committees of Boston University (14-008) and the

169 Smithsonian Tropical Research Institute (2014-0601-2017).

\section{Measurement of the vestibulo-ocular reflex (VOR)}

172 We measured roll-induced VOR of newly hatched tadpoles or manually decapsulated

173 embryos (henceforth, collectively 'hatchlings') using a custom-built, Arduino-based, portable

174 tadpole rotator (Fig. S1, J. G. McDaniel, Adrian Tanner, and K. M. Warkentin; Boston

175 University Engineering Products Innovation Center). The rotator smoothly turns a shaft at the

176 push of a 'clockwise' or 'counterclockwise' button and was programmed for $15^{\circ}$ rotational

177 increments. A conditioning mass and rubber plate mounted on the shaft limit vibration transfer

178 from the motor to the test animal, and a printed plastic cup glued to the rubber plate enables field

179 replacement of the animal interface. To hold hatchlings, we mounted a section of plastic pipette

180 in the center of the cup, in line with the rotator shaft, using silicone seal. The hatchling chamber

181 was $13.5 \mathrm{~mm}$ long and $3 \mathrm{~mm}$ in internal diameter, with a slight widening at the mouth so as not

182 to restrict eye motion, and horizontally leveled in relation to gravity. To test a hatchling, the

183 chamber was filled with aged tap water and the animal was backed into it using a transfer pipette

184 or length of tubing on a syringe, positioning its snout just within the tube. No anesthesia was

185 necessary and individuals could be tested within minutes of hatching.

186 The chamber was surrounded by a light diffuser and illuminated on both sides by LED

187 lights (Panasonic 9W, 100-127V, 90mA), providing a uniform white visual field. It faced a

188 horizontally leveled MPE-65 mm macro lens on a digital camera (Canon D70) with cable shutter

189 release, mounted on a focusing rail on a tripod. Following Horn (Horn et al., 1986b; Horn and 
190 Sebastian, 1996), we rolled hatchlings about their body axis $180^{\circ}$ in each direction,

191 photographing them in frontal view each $15^{\circ}$ (Movie S1). We continuously observed hatchlings

192 on the camera view-screen, manually applied rotation increments, and took each photograph as

193 soon as body and eye rotation had stopped, to minimize testing time. Most animals remained

194 immobile through each $180^{\circ}$ roll sequence; for those that moved more than their eyes, we

195 restarted the sequence from $0^{\circ}$ to obtain a continuous series of measurements. From each

196 photograph, we measured right and left eye angle and body axis angle using ImageJ (Schneider

197 et al., 2012). From each angular measurement series, we constructed an individual VOR curve

198 using a sine-fitting function in Python (Version 2.7.9, Build 1, Python Software Foundation). We

199 assessed the curve fit and calculated the VOR amplitude from the sine function. The peak-to-

200 peak amplitude of the curve corresponds to the hatchling's VOR magnitude (Fig. 1).

201 We visually checked each sine curve fit and rejected those that did not meet the following

202 criteria: 1) curve fits of the two eyes show similar wavelengths, are horizontally aligned, and

203 have parallel or near-parallel waveforms, 2) the wavelength is plausible for VOR, with zero

204 crossing at or near the zero body angle, and 3) eye rotation is opposite to body rotation (i.e.,

205 curve is not upside-down). Individuals whose curve fits failed one or more of these VOR criteria

206 (Fig. S2) were considered to have a VOR of zero. Of 406 hatchlings tested, 92 failed the VOR

207 curve fit criteria ( $\mathrm{N}=4$ of 36 in series Ia below, $\mathrm{N}=38$ of 89 in Ib, N = 19 of 169 in II, N = 31

208 of 112 in III).

209

210 I. Ontogeny of vestibular function

211 First, to determine the basic ontogenetic timing of the onset of vestibular sensory function

212 in A. callidryas, we measured the VOR of embryos at different ages (Ia). From 19-25 June 2014,

213 we tested VOR daily across the plastic hatching period, in the afternoon of each day (13:27-

214 17:09 h), using a set of non-sibling embryos at each age ( $\mathrm{N}=7,10,10$, and 9 hatchlings, at ages

215 3-6 d respectively; total $\mathrm{N}=36$ hatchlings from 14 clutches). Second, to assess how VOR varied

216 among and within egg clutches across development, we tested developmental series of five

217 clutches, from 10-20 August 2014 (Ib). We concentrated our sampling in the period of greatest

218 change, testing $\sim$ three siblings per age at $6 \mathrm{~h}$ intervals from 3.75-4.75 d, with a final sample at

$2195.75 \mathrm{~d}$ (total $\mathrm{N}=88$ hatchlings; $\mathrm{N}=15,16,15,15,15,12$ individuals per age group). 
We removed each individual egg from its clutch just prior to VOR testing, placed it in a small dish, and gently rolled and jiggled it with a blunt probe to induce hatching. The youngest embryos were unresponsive to this stimulus and, instead, manually decapsulated with fine

223 forceps under a dissecting microscope. Hatchlings were tested for VOR within 3 minutes of

224 leaving their egg capsule.

\section{VOR and hatching response in vibration playback to whole clutches}

To assess if variation in VOR and the hatching response are related, across the period of high variation in both traits, we paired vibration playbacks to 36 clutches with VOR measurements on a subset of embryos from each clutch, from 26 June to 21 July 2015 . We tested clutches at ages 3.7-4.9 $\mathrm{d}$ and stages 1-7 (Warkentin et al., 2017), from before any hatching response to vibration until responses became fairly strong. To focus on fine-scale developmental

232 changes and avoid age-imprecision due to variation in oviposition timing, we report results based 233 on stage. Compared to 2014, development tended to be accelerated under the warm El Niño 234 conditions in 2015 (Warkentin et al., 2017).

235 We played a synthetic low-frequency vibration stimulus (Fig. 2A) designed to elicit very 236 high hatching, based on prior playbacks to 5-d-old clutches (Caldwell et al., 2009; Warkentin et 237 al., 2006b). We generated noise in Matlab and filtered it using a custom script, playback.m 238 (available upon request), to compensate for nonlinearities in the shaker transfer function and 239 generate a frequency distribution resembling that of snake attacks (Caldwell et al., 2009), with 240 high energy below $60 \mathrm{~Hz}$ and intensity dropping off above that (Fig. 2C). To test our match to

241 the desired frequency distribution, we recorded playbacks of the stimulus embedding a small

242 (0.14 g) AP19 accelerometer (AP Technology International B.V., Oosterhout, The Netherlands)

243 within a clutch. Accelerometers added $\sim 5 \%$ to the mass of each clutch, such that test clutches

244 remained within the natural range of interclutch mass variation (Warkentin 2005). Transduced

245 vibrational signals were powered/amplified by an APC7 signal conditioner and digitized with an

246 external sound card (MSE-U33HB; Onkyo USA, Saddle River, NJ, USA). The output was

247 recorded using Raven Pro 1.3 bioacoustics software (Cornell University Laboratory of

248 Ornithology, Ithaca, NY, USA) on a Macbook Pro computer. The intensity of frequencies below

$24920 \mathrm{~Hz}$ was limited by shaker capabilities. The base temporal pattern consisted of $0.5 \mathrm{~s}$ pulses of

250 vibration, with roughly rectangular amplitude envelopes, separated by $1.5 \mathrm{~s}$ intervals of silence 
251 (Fig. 2B). This was divided into pulse-groups consisting of 10 pulses separated by 30-s gaps of

252 silence (Fig. 2B). We included a three-pulse "primer" plus 30-s gap before the repeating10-pulse

253 pattern began, since this element also increases hatching response (Fig. 2B, Jung, Guo, McDaniel

254 and Warkentin, unpublished data).

255 Playback methods followed Caldwell et al. (Caldwell et al., 2009; Caldwell et al., 2010).

256 Stimuli (Fig. 2A) were presented through an array of blunt metal tines inserted among eggs (Fig.

257 S3, Movie S2) attached via a rigid post to an electrodynamic minishaker (Model 4810; Brüel \&

258 Kjær, Nærum, Denmark). Shaker output was controlled by Audacity 2.1.0 (Free Software

259 Foundation, Boston, MA) on a 2014 MacbookAir, via a custom-made amplifier designed to have

260 a flat frequency response from DC to $5 \mathrm{kHz}$ (E. Hazen, Boston University Electronic Design

261 Facility). Playback clutches on their plastic cards were mounted on a flat-sided plastic stand

$262(\sim 1.5 \mathrm{~kg})$, then carefully slid forward so the tines entered the clutch between eggs. We used only

263 healthy clutches that fit within the tine field, and tines were rinsed with rainwater between trials.

264 We watched for any hatching induced by the set-up procedure (only 3 individuals, from 3

265 clutches), then allowed five hatching-free minutes for acclimation before starting the playback.

266 For playback, the shaker moved the tines up and down, so eggs were shaken vertically, and

267 hatched tadpoles fell into a tray of water below the clutch.

$268 \quad$ For each trial, we counted the embryos that hatched during the playback period and 5-

269 min of post-playback observation. We then immediately (within 5-10 minutes) measured VOR

270 of a subset of 3 hatchlings per clutch that had hatched in response to playback, unless fewer had

271 hatched. To check for hatching competence of the remaining eggs, after post-playback

272 observation, we manually stimulated eggs, rubbing and jiggling them with a blunt metal probe,

273 for about two minutes, then submerged any unhatched eggs in hypoxic water. Any embryos that

274 failed to hatch under manual stimulation and hypoxia were considered not competent to hatch,

275 and excluded from the count of test individuals in calculations of proportion hatched per clutch

276 (proportion excluded $=0.078 \pm 0.022$, mean \pm s.e.m. across clutches). We measured VOR of 3

277 additional hatchlings that hatched in response to either manual stimulation or hypoxia, but not

278 vibration playback; numbers of manually-stimulated and hypoxia-cued hatchings tested for VOR

279 varied among clutches (total of 3, unless fewer remained after playback). We staged all VOR-

280 tested hatchlings $(\mathrm{N}=143$ hatchlings total) from their frontal photos following a staging system

281 adapted from Warkentin et al. (2017) (Fig. 3). 


\section{VOR and hatching response to simulated attack on individual embryos}

To examine the correlation between hatching responses to physical disturbance cues and vestibular function on an individual level, we assessed both traits in developmental series of embryos across the onset of mechanosensory-cued hatching. To assess hatching responses of embryos to a simulated attack, we removed individual eggs from their clutch, placed each in a petri dish with a drop of water, and manually jiggled them with a moistened blunt metal probe, alternating $15 \mathrm{~s}$ of stimulation and $15 \mathrm{~s}$ of rest for $5 \mathrm{~min}$ or until the egg hatched (Warkentin et al., 2017) (Movie S3). We tested two embryos per clutch from 11 clutches every 3 hours, on

291 August 11-13, 2015. As with vibration playbacks, we observed embryos for 5 min before, 292 during, and after stimulation (15 minutes total), and considered any hatching during and after 293 stimulation (10 minutes) to be a response to the stimulus. All sibships were initially tested for

294 their hatching response to hypoxia and, in most cases, we began testing responses to the egg-

295 jiggling stimulus only after siblings had demonstrated an ability to hatch; the data on

296 developmental timing of onset of the response to each cue are reported elsewhere (Warkentin et

297 al., 2017). We continued testing each clutch every $3 \mathrm{~h}$ until both test embryos had hatched at two

298 time points, thus capturing a range of developmental ages (3.25-4.625 d) and stages (2-7) from

299 those unresponsive to the jiggling cue, through the onset of response, to strongly responsive

300 (total $\mathrm{N}=112$ individuals, 6-18 per clutch). For each hatchling we recorded latency to hatch,

301 from stimulus onset, or failure to hatch after $5 \mathrm{~min}$ of post-stimulus observation. We manually

302 decapsulated unhatched embryos, and photographed all animals in frontal view to assess

303 development (from stages 2-8) following a staging system adapted from Warkentin et al. (2017)

304 (Fig. 3). We then immediately measured their roll-induced VOR.

Statistics

When data met parametric assumptions, we used ANOVAs and Tukey post-hoc tests to

308 find effects and comparisons. Otherwise, we used the Wilcoxon Rank Sum and Wilcoxon Each

309 Pair methods for non-parametric tests of effects and comparisons. In the first developmental

310 assay that examined the hatching response of multiple siblings per clutch (Ib), we fit a 4-

311 parameter logistic model, grouped by clutch, and performed an analysis of means for inflection

312 point estimates. In the following assays where we considered multiple siblings per clutch, we 
313 analyzed our results using mixed models with clutch as a random effect. To analyze predictors of

314 hatching, we used binomial GLMMs with clutch as a random effect and performed likelihood

315 ratio tests to compare nested models. All statistical tests were carried out in JMP Pro 13 (version

316 13.2.0, SAS Institute Inc. 2016) or the R statistical environment (version 3.3.3, R Development

317 Core Team 2014, http://www.r-project.org) in RStudio (version 1.1.383, RStudio Team 2015).

\section{RESULTS}

\section{Ontogeny of vestibular function}

322 Across embryos tested at daily intervals, VOR amplitude increased with age (Wilcoxon

323 Rank Sum: $\chi^{2}=16.2797, \mathrm{df}=3, \mathrm{P}=0.0010$, Fig. 4A). VOR did not change significantly from age

324 4-6 d (Wilcoxon Each Pair, all P>0.4274) but hatchlings tested at age $3 \mathrm{~d}$ showed lower VOR

325 than those aged 4-6 d (Wilcoxon Each Pair, all $\mathrm{P}<0.0014$, Fig. 4A). In the second developmental

326 series, with replication within clutches, VOR increased with age in a sigmoidal fashion $\left(\mathrm{R}^{2}=0.91\right.$,

327 Fig. 4B), and clutches varied in inflection point estimates (Analysis of means; upper limit

328 exceeded in clutch 101 and 102, $\mathrm{P}<0.01$; Fig. 4B).

\section{VOR and hatching response in vibration playback to whole clutches}

331 Based on post-playback hypoxia testing, all individuals included in VOR analyses

$332(\mathrm{~N}=169)$ were able to hatch, but only 63 of them hatched in response to vibration playbacks.

333 VOR amplitude increased significantly across developmental stages (one-way ANOVA,

$334 \mathrm{f}_{5,162}=79.2953, \mathrm{P}<0.0001$ ). Across the first four stages we tested (stages 2-5, Warkentin et al.

335 2017), no embryos in any clutches hatched in response to vibration playbacks and VOR was

336 consistently low $\left(5.3 \pm 1.0^{\circ}\right.$, mean \pm SE, here and throughout; N=22 hatchlings, 8 clutches; Fig.

337 5A). Compared to VOR at stages $2-5$, VOR was higher at stage 6 ( $N=63$ hatchlings, Tukey test

338 from one-way ANOVA, $\mathrm{P}<0.0001)$ and stage $7(\mathrm{~N}=84$ hatchlings, $\mathrm{P}<0.0001$, Figure $5 \mathrm{~A})$. Up

339 until stage 5, no individuals hatched. At stage 6, vibration-cued hatching began, but the low

340 hatching response rates within clutches were not significantly higher than zero at earlier stages

341 (Tukey test from one-way ANOVA, P>0.3809); clutch hatching rates at stage 7 were

342 significantly higher than at all prior stages $(\mathrm{P}<0.0001)$. 
344 higher VOR than individuals that did not hatch in playback, but hatched in response to manual

345 stimulation or hypoxia (Mixed Model, VOR Amplitude $\sim$ Hatching with Clutch as a random

346 effect: $\left.\chi^{2}=4.8028, \mathrm{P}=0.02841\right)$. For the subset of 23 clutches where hatching occurred,

347 individuals that hatched in playbacks tended to have higher VOR than siblings that did not hatch,

348 but the difference was not significant (Mixed Model, $\chi^{2}=3.0898, \mathrm{P}=0.07878$ ). However,

349 individuals that hatched only in response to hypoxia had significantly lower VORs than those

350 that hatched in response to playbacks (Mixed Model, $\chi^{2}=6.2563, \mathrm{P}=0.0438$ ). For clutches with

351 hatching, we compared mean VOR of each clutch (from 5.61 \pm 0.14 individuals per clutch, range

352 4-6) with proportion hatched. Proportion hatched per clutch increased with mean VOR (Figure

$3535 \mathrm{~B}$, linear regression, $\mathrm{F}_{1,21}=8.0252, \mathrm{P}=0.0100$ ); no individuals hatched with VOR less than

$35421.38^{\circ}$ and mean VOR of those that hatched in response to playback was $35.92^{\circ} \pm 0.95^{\circ}$.

\section{VOR and hatching response to simulated attack on individual embryos}

For individual embryos $(\mathrm{N}=112)$ subjected to a simulated attack, VOR increased in magnitude across embryonic developmental stages (Mixed Model, $\chi^{2}=96.215, \mathrm{P}<2.2 \mathrm{e}-16$, Fig. $6 \mathrm{~A}$ ) and varied among clutches (Mixed Model, $\chi^{2}=8.3355, \mathrm{P}=0.003888$ ). No stage 2 embryos hatched. Hatching in response to individual egg-jiggling began at stage 3 with a hatching rate of 20.8\% (Fig. 6B), which is when some embryos started showing a measurable VOR (Fig. 6A). By stage 4 , almost half the embryos hatched (47.4\%) and by stage 7, all embryos tested hatched in response to the jiggling cue (Fig. 6B).

Both developmental stage and VOR amplitude were significant and strong predictors of 365 hatching (Fig. 7A-B, binomial GLMM), and the model incorporating both variables was better 366 than models with either one alone (AIC values 120 vs. 122 and 125). More developed embryos 367 with greater VOR were more likely to hatch, with hatching response increasing 14\% for every 368 stage $\left(\chi^{2}=13.285, \mathrm{P}=0.02085\right)$ and $24 \%$ for every 10 degrees of VOR amplitude $\left(\chi^{2}=11.951\right.$, $369 \mathrm{P}=0.0005461$, Fig. 7A-B). However, the 61 embryos that hatched in response to egg-jiggling included 7 individuals with no detectable VOR, ranging from stage 3 to 5 (Fig. 8).

372 to hatch decreased with VOR amplitude (Fig. 8, Latency $\sim$ VOR with Clutch as a random effect,

$\left.373 \chi^{2}=16.55, \mathrm{P}=4.738 \mathrm{e}-5\right)$. If we add stage and the interaction between stage and VOR into the 
374 model, there is a main effect of stage $\left(\chi^{2}=13.3925, \mathrm{P}=0.009509\right)$, and an interaction effect

$375\left(\chi^{2}=12.0126, \mathrm{P}=0.017258\right)$, but no main effect of $\operatorname{VOR}\left(\chi^{2}=1.3143, \mathrm{P}=0.251613\right)$. Closer

376 examination of the interaction indicates a significant VOR effect only at stage $6\left(\chi^{2}=5.0734\right.$,

$377 \mathrm{P}=0.0243$ ), but note that sample sizes were lower at other stages (in order from stage 3, N=5, 9,

$37818,26,3)$.

379

\section{DISCUSSION}

381

382

Embryos use physical disturbance (egg motion) as a cue to hatch among fishes (Martin et

384 2018; Touchon et al., 2011; Warkentin, 1995; Warkentin, 2000; Warkentin, 2011b), and reptiles

385 (Doody, 2011; Doody and Paull, 2013; Doody et al., 2012), as well as many invertebrates (Endo

386 et al., 2018; Mukai et al., 2014; Oyarzun and Strathmann, 2011; Tanaka et al., 2016; Whittington

387 and Kearn, 1988). However, the specific sensors mediating the environmentally cued hatching

388 responses of embryos are entirely unknown. We examined the role of the vestibular system - the

389 general vertebrate motion sensor - in the escape-hatching response of red-eyed treefrogs. In four

390 experiments, at population, clutch, and individual levels, we found developmental congruence

391 between the onset of the VOR and the escape-hatching response to real and simulated predator

392 attack and vibration playbacks, consistent with our hypothesis that the vestibular system plays a

393 key role in mediating mechanosensory-cued hatching.

394

\section{VOR as an indicator of vestibular system function}

396 Our tests for ontogenetic congruence of vestibular system function and escape-hatching

397 behavior are based on the vestibulo-ocular reflex (VOR), or eye movements induced by roll and

398 tilt of the body (Horn et al., 2013), which we could measure within minutes of hatching using a

399 tadpole-in-tube rotation protocol. Since input from the vestibular system controls the muscles

400 responsible for VOR, it is well-established that the VOR is not expressed without vestibular

401 system function (Cohen, 1974; Precht, 1976). Moreover, the onset of VOR appears not to be

402 limited by eye muscle development. Extraocular motoneurons develop and establish axonal

403 connections with target eye muscles very early in embryogenesis (Gilland and Baker, 2005;

404 Glover, 2003). In 96 of 406 hatchlings tested, we observed non-VOR-related eye movements 
405 (criteria listed in methods) with a measurable magnitude greater than that of individuals with a

406 small but clear VOR (Fig. S2). This indicates that hatchlings, prior to developing a working

407 VOR, can change their eye angle - just in a way that does not match up with their body rotation.

408 The data from these individuals supports that the onset of VOR is not limited by when embryos

409 become physically capable of moving their eyes. Moreover, the presence of non-VOR-related

410 eye movements motivate our criterion rejecting individuals with non-parallel curve fits. The eye

411 muscles that enable the VOR receive their information from both vestibular organs (Precht,

412 1976). In Xenopus, complete unilateral vestibular lesions and selective lesions of each utricular

413 organ reduce the VOR of both eyes (Horn et al., 1986b). Thus, we considered non-parallel

414 curves for the two eyes to indicate non-VOR-related eye movements (Fig. S2A).

416 Ontogenetic congruence of VOR and mechanosensory-cued hatching

417 When we began this work, we knew that hatching ability does not limit the onset of

418 hatching responses to predator cues, because younger embryos demonstrate hatching competence

419 in response to strong hypoxia (Cohen et al., 2019; Warkentin et al., 2017). Moreover, the rapid

420 developmental increase in hatching response to egg-jiggling - contrasted with the much slower

421 developmental decrease in the costs of early hatching - suggests that some sensory constraint

422 imposes a developmental limit on the onset of the anti-predator response (Warkentin et al.,

423 2017). We performed four experiments to examine the role of vestibular mechanoreception in

424 embryos' risk assessment by comparing the ontogeny of responses at a population level, at a

425 clutch level, and at an individual level.

426 First, at a population level, we found that the developmental onset of the vestibulo-ocular

427 reflex in red-eyed treefrog embryos (individually in series Ia and across clutches in series Ib) is

428 congruent with the documented onset of escape-hatching responses to predator attacks in the

429 Gamboa population of $A$. callidryas (Almanzar and Warkentin, 2018; Hite et al., 2018;

430 Warkentin, 2000; Warkentin et al., 2006a). If the onset of VOR were clearly before or after the

431 developmental period when predator-induced hatching begins in this population of A. callidryas,

432 it would have rejected the hypothesized key role of vestibular system development in enabling

433 the antipredator response. Moreover, clutches appeared to vary slightly in their onset of VOR

434 (Ib), congruent with the greater variation in escape-hatching success of clutches attacked when 
435 the response first appears, and decreased variation later in development (Gomez-Mestre et al., 436 2008; Warkentin et al., 2006a).

437 Next, we examined the ontogeny of VOR in more detail through the period of greatest

438 change (series II) and tested its relationship to the hatching response using vibration playbacks to 439 entire clutches. Across the onset of ear function, embryos below a VOR threshold of $21^{\circ}$ did not

440 hatch during vibration playbacks, even though they could hatch if flooded. Moreover, clutch

441 hatching response increased with clutch mean VOR at supra-threshold levels. These data are also 442 consistent with a key role of vestibular mechanosensing in mediating vibration-cued hatching.

443 In our last series (III), we compared VOR and the hatching response to simulated attacks

444 on individual embryos, rather than whole clutches, and saw that they were still highly correlated.

445 In addition, embryos with greater VOR hatched more rapidly in response to egg jiggling.

446 Hatching occurred developmentally earlier in response to targeted jiggling cues (III) than in

447 response to whole-clutch vibration playback (II), at stage 3 vs. stage 6. Moreover, embryos

448 started showing a measurable VOR at earlier developmental stages in the egg jiggling series

449 (III), relative to the clutch vibration series (II) (compare Fig. 5A vs. 6A). VOR development was

450 correlated with stage, but not perfectly. For instance, some stage 3 animals showed VOR but

451 most did not, and one stage 5 animal lacked VOR, but most showed it. VOR amplitude predicted

452 hatching more strongly than did developmental stage although, controlling for VOR, stage

453 explains some additional variation and vice-versa. This individual-level correlation between

454 vestibular function and hatching is consistent with a role of the vestibular system in

455 mechanosensory-cued hatching.

456 Across successively finer levels of developmental precision, our results reveal a

457 substantial increase in mechanosensory-cued hatching responses with the development of

458 vestibular function, consistent with a role for this sensory system in mediating the response. In

459 general, the timing of onset of vestibular function is consistent with the onset of escape success

460 in predator attacks (Almanzar and Warkentin, 2018; Hite et al., 2018; Warkentin, 2000;

461 Warkentin et al., 2006a). In our vibration playbacks to clutches, no embryos lacking VOR

462 hatched. In our egg-jiggling developmental series, we found a strong correlation of VOR with

463 increased hatching response and decreased hatching latency. However, some evidence suggests

464 that additional mechanoreceptor systems can also play a role in escape-hatching (Fig. 7B). 


\section{Mechanosensory-cued hatching before vestibular function}

Of the 61 embryos that hatched in response to our individual egg-jiggling cue, seven

468 individuals (11\%) had no detectable VOR; they hatched an average of $4.85 \mathrm{~h}$ before their

469 siblings showed VOR. Hatching of embryos lacking VOR in response to jiggling cues is

470 relatively rare and does not reject a key role of the vestibular system in risk assessment by

471 embryos, given the strength of the relationship between VOR and hatching. However, the

472 occurrence of any mechanosensory-cued hatching prior to vestibular function indicates that

473 vestibular mechanoreceptors are not the only sensors that can mediate hatching when eggs are

474 physically disturbed, at least under some types of disturbance. A. callidryas embryos clearly use

475 cues in multiple sensory modalities, including hypoxia (Rogge and Warkentin, 2008) and light

476 level (Güell and Warkentin, 2018), to inform hatching. These embryos might also use multiple

477 mechanosensors, either to perceive different cue components available in attacks and egg-

478 jiggling or as potentially redundant or synergistic sensors of the same cue element. Two other

479 candidate sensor types - lateral line neuromasts and cutaneous mechanoreceptors - may also be

480 relevant to mechanosensory-cued hatching in the egg-jiggling context.

\section{Other mechanosensory systems}

483 The lateral line is a system of mechanoreceptors that detect movement, pressure

484 gradients, and vibration in fishes and aquatic amphibians (Mogdans and Bleckmann, 2012). The

485 effective stimulus to lateral line is low frequency particle motion of the surrounding fluid,

486 relative to neuromasts distributed on the animal's surface (Strelioff and Honrubia, 1978; Weeg

487 and Bass, 2002). A. callidryas embryos develop a lateral line system on their head, body, and tail

488 by $3 \mathrm{~d}$, well before mechanosensory-cued hatching begins at $4 \mathrm{~d}$ (Cohen et al., 2019; Warkentin

489 et al., 2017). However, the number of superficial neuromasts, visualized with the fluorescent

490 vital dye DiAsp (Sigma D-3418), continues to increase through the onset of mechanosensory-

491 cued hatching (Jung and Warkentin, unpublished data). The constant ciliary circulation of the

492 perivitelline fluid within $A$. callidryas eggs (Rogge and Warkentin, 2008; Warkentin et al., 2005)

493 presumably stimulates the lateral line, and any change in this circulation pattern would therefore

494 be perceptible to embryos.

495 The sensation of touch in adult frogs and tadpoles depends on cutaneous

496 mechanoreceptors that are diverse and highly specialized (Catton, 1976; Fromy et al., 2008; 
497 Spray, 1976; Weston, 1970). A single mechanoreceptive afferent can encode more than one type

498 of stimulus, for example temperature and texture (Hunt and McIntyre, 1960), as well as

499 mechanical stimuli such as pressure and vibration (Ribot-Ciscar et al., 1989). Since all

500 somatosensory neurons arise from precursor neural crest cells early in embryonic development,

501 much prior to the development of the vestibular system (Jenkins and Lumpkin, 2017; Weston,

502 1970), pre-VOR A. callidryas embryos are likely to already have cutaneous mechanoreceptors.

503 These could enable embryos to sense contact cues, through the membrane, as a probe or a

504 predator touches the egg capsule. Moreover, if the inertia of embryos is higher than their

505 surroundings, moving an egg might also change how strongly the embryos' skin presses against

506 the adjacent membrane, altering contact cues.

507

508

509

510

511

512

513

514

515

516

517

518

519

520

521

522

523

524

525

526

\section{Multiple mechanosensory cues in attacks and multiple mechanosensory systems}

Several types of mechanosensory cues could occur in egg-predator attacks - and in eggjiggling - including whole-egg motion, embryo motion within the capsule, and tactile contact that may deform egg-capsules or contact embryos through their perivitelline membranes (Fig. 9). Whole-egg motion occurs in vibration-playbacks, egg-jiggling, and predator attacks. This will activate the vestibular system as the embryo is passively accelerated along with its surrounding capsule. If the embryo remains in the same position relative to its capsule, whole-egg motion alone would likely not alter perivitelline fluid flow and seems unlikely to stimulate the lateral line or cutaneous touch receptors.

Embryo motion within the capsule occurs when embryos are displaced in their perivitelline chamber as the capsule is moved. The inertia of the embryo likely differs from the surrounding fluid and capsule, such that the embryo may lag a bit behind as the egg accelerates around it. For instance, if the egg were accelerated up the embryo could be pressed against the bottom of the chamber, and if the egg were accelerated down the embryo could be lifted off the bottom. This could change both cutaneous stimulation and perivitelline fluid flow if the embryo's body were sufficiently displaced within the capsule. Tactile contact occurs for a subset

524 of eggs in predator attacks on, and tine-based vibration playbacks to, whole egg clutches, and for 525 all eggs exposed to individual egg-jiggling stimulation. If the contact deforms egg capsules (e.g., dents or squashes them), even without contacting the embryo inside, it may change perivitelline 
527 fluid flow and lateral line input (Fig. 9). Contact with the embryos through the membrane would 528 also directly stimulate cutaneous touch receptors.

529 Our egg-jiggling and vibration-playback experiments differed in several important ways.

530 First, the jiggling stimulus represents a targeted attack on individual eggs rather than a

531 generalized stimulus to whole clutches. Second, it was a more complex multimodal stimulus that

532 combined whole egg motion with tactile elements and included both lateral and rolling

533 movements. Since predators must touch eggs to eat them, risk of mortality in attacks is

534 presumably higher for eggs receiving motion and contact cues than for those receiving motion

535 cues alone. Both targeted jiggling of and predator attacks on individual eggs likely stimulate the

536 vestibular system, the lateral line, and touch receptors in the skin. But other eggs in attacks and

537 in vibration playbacks likely experience only whole-egg motion and vestibular stimulation. This

538 variation in the cues available to embryos may contribute to the variation in individual responses

539 and the different responses to vibration-playback and egg-jiggling stimuli. Moreover, in the

540 jiggling series, embryos began showing VOR (thus, developing vestibular function) at earlier

541 developmental stages compared to in the tine playback series (Fig. 6A vs. 5A), which may also

542 have contributed to their earlier mechanosensory cued hatching.

543

544 Ontogenetic changes in embryo use of multimodal mechanosensory cues

$545 \quad$ Whatever sensory system mediates hatching in egg jiggling for animals lacking VOR

546 would presumably add to the stimulation experienced by older animals that have developed a

547 functional vestibular system. Moreover, at a given stage of development, cues indicating greater

548 risk should be more likely to elicit hatching. Thus, at the same stage, we expect an individually

549 targeted "attack" stimulus to more strongly elicit hatching than a stimulus transmitted through

550 the clutch. Consistent with this, stage 6 or 7 animals with strong VOR show a stronger hatching

551 response to egg jiggling than to vibration playbacks to clutches. Nonetheless, if an animal has

552 less-developed mechanoreceptors and cannot sense components of a stimulus, it will be limited

553 in its risk-assessment ability. Stage 3 animals lacking VOR, and vestibular function, presumably

554 receive just cutaneous and perhaps lateral line input in attacks and our mechanosensory stimuli.

555 In contrast, stage 3-5 animals with low VOR likely also receive weak vestibular input;

556 combining this with cutaneous and/or lateral line input may generate sufficient total stimulation

557 to elicit hatching. Without additional input from another mechanosensory system, weak 
558 vestibular input may be insufficient to elicit hatching. Different types of mechanosensory cues

559 likely stimulate different mechanoreceptor types, or combinations thereof, providing different

560 and potentially synergistic or complementary information about risk. Thus, A. callidryas

561 embryos may use multimodal mechanosensory cues to inform escape-hatching decisions,

562 particularly at the onset of vibration-cued hatching when their mechanosensory systems are less

563 developed.

564 We recently developed a new vibration playback system to generate whole-egg motion

565 without tactile contact cues or egg-shape deformation, demonstrating that egg-motion alone is

566 sufficient to induce hatching (Warkentin et al., 2019). A second playback-system component

567 adds a tactile contact cue, which appears to synergize with motion to increase hatching of 4-day

568 embryos (Fouilloux, Jung, Ospina, Snyder, \& Warkentin unpublished). Lateral line blocking

569 and/or vestibular system ablation experiments, in conjunction with vibration playbacks, would be

570 useful to assess the individual and potentially interacting roles of these mechanosensory systems

571 in the hatching decisions of $A$. callidryas embryos.

572

\section{Conclusion}

574 Hatching is a developmentally critical behavior that immediately impacts survival in

575 multiple ecological contexts. Environmentally cued hatching is widespread and well-documented

576 in all three major clades of bilateria and, in many species, embryos respond to multiple different

577 factors or contexts (Warkentin, 2011a). Physical disturbance of eggs is a particularly salient and

578 common cue to hatch among embryos of fishes (Martin et al., 2011), amphibians (Buckley et al.,

579 2005; Gomez-Mestre et al., 2008; Goyes Vallejos et al., 2018; Touchon et al., 2011; Warkentin,

580 1995; Warkentin, 2000; Warkentin, 2011b), and reptiles (Doody, 2011; Doody and Paull, 2013;

581 Doody et al., 2012), as well as many invertebrates (Endo et al., 2018; Mukai et al., 2014;

582 Oyarzun and Strathmann, 2011; Tanaka et al., 2016; Whittington and Kearn, 1988). Presumably,

583 all the vertebrates that use physical disturbance as a hatching cue have vestibular systems and

584 cutaneous mechanoreceptors, but only the fish and amphibians have lateral lines. Moreover,

585 some of the contexts that induce hatching in vertebrates seem likely to provide only whole-egg

586 motion cues. For instance, grunion embryos are tightly coiled within their eggs and pressed

587 against the capsule wall at a stage when tumbling in waves elicits hatching (Martin et al., 2011;

588 Speer-Blank and Martin, 2004). This embryo size and position seem likely to prevent passive 
589 displacement within the perivitelline chamber as eggs are moved. Pig-nosed turtle embryos hatch

590 in response to a whole-egg motion stimulus presented via an electronic shaker in the laboratory

591 (Doody et al., 2012). Neither the lateral line nor cutaneous sensing seem likely to play a role in

592 these instances, suggesting the vestibular system could mediate motion-cued hatching responses

593 in multiple - perhaps many — vertebrate embryos. The mechanisms that enable, regulate, and

594 inform hatching change developmentally, altering embryos' capacities for behavioral responses

595 to cues. Thus, information on embryos' sensory development will clarify how and why

596 development changes behavior. This research elucidates how changing sensory and behavioral

597 abilities can affect an essential early behavior and reveals a fundamental mechanism underlying

598 phenotypic plasticity at a critical life history switch point.

599

Acknowledgments

601 This research was funded by the Smithsonian Tropical Research Institute, the National

602 Science Foundation (IOS-1354072 to K.M.W. and J.G.M.) and Boston University, including

603 grants from BU's Undergraduate Research Opportunity Program to S.M.P.A. and S.J.K. It was

604 conducted under permits SC/A-15-14 and SE/A-46-15 from the Panamanian Ministerio de

605 Ambiente, STRI IACUC protocol 2014-0601-2017, and BU IACUC protocol 14-008. We thank

606 Adrian Tanner for helping design and build the tadpole rotator, Nora Moscowitz and Angelly

607 Vasquez for help with egg care in 2015, and Alina Chaiyasarikul and Adeline Almanzar for

608 assistance measuring images to calculate VOR. We thank members of the Gamboa Frog Group

609 at STRI and BU Egg Science Research Group for discussions of this research at many stages of

610 its development.

611

612 Competing Interests

613 No competing interests declared.

614

615 Author Contributions

616 J.G.M. and K.M.W. designed and built the tadpole rotator and J.G.M. provided vibrations

617 engineering support for playbacks. S.M.P.A. and K.M.W. designed, and S.M.P.A. conducted,

618 experiments Ia and Ib. J.J., S.J.K., and K.M.W. designed, and J.J. and S.J.K. conducted, 
bioRxiv preprint doi: https://doi.org/10.1101/634899; this version posted May 10, 2019. The copyright holder for this preprint (which was not certified by peer review) is the author/funder. All rights reserved. No reuse allowed without permission.

619 experiments II and III. S.M.P.A, J.J. and S.J.K. measured VOR for all experiments. J.J. analyzed 620 the data from all experiments. J.J. wrote the paper. All authors edited the paper. 

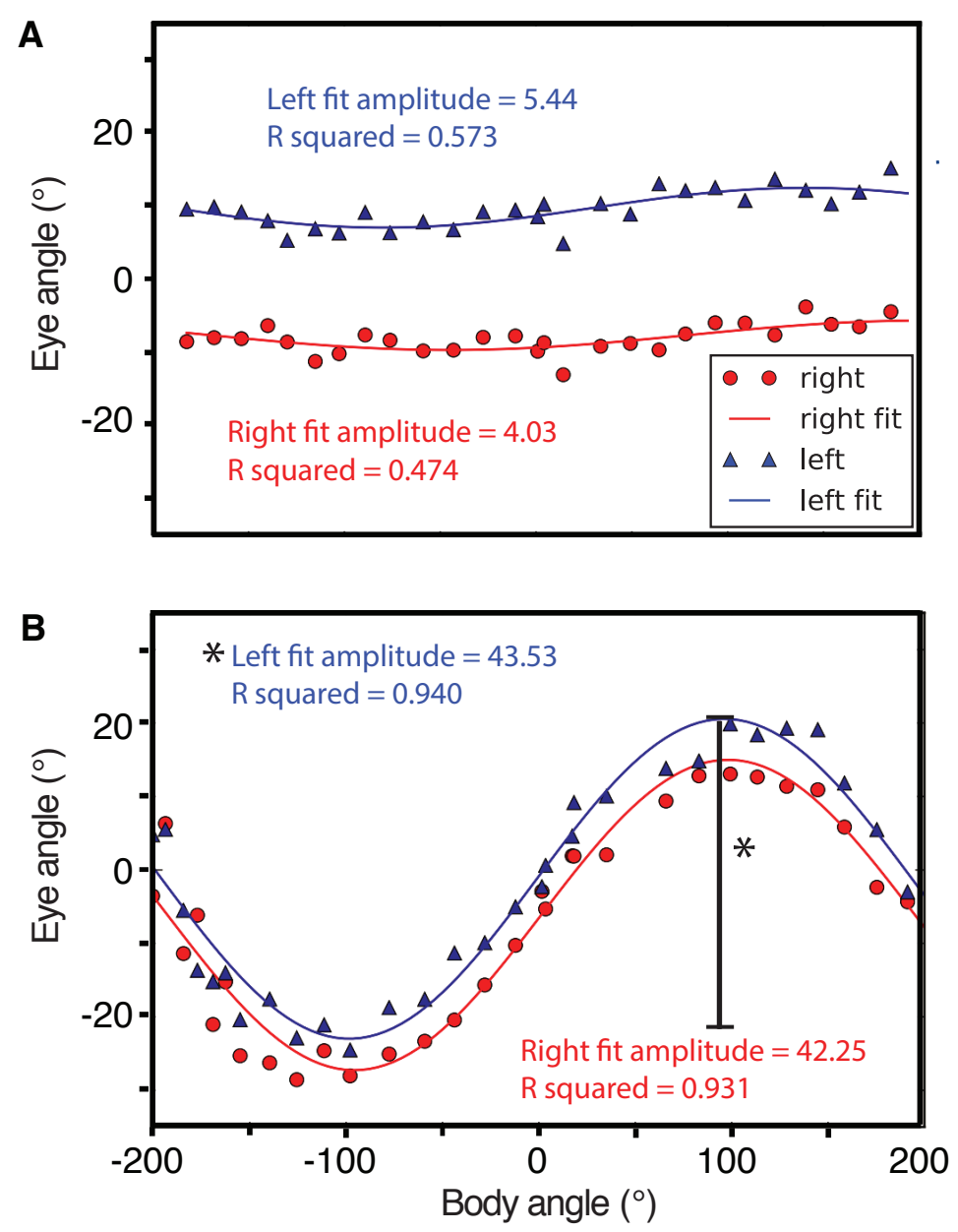
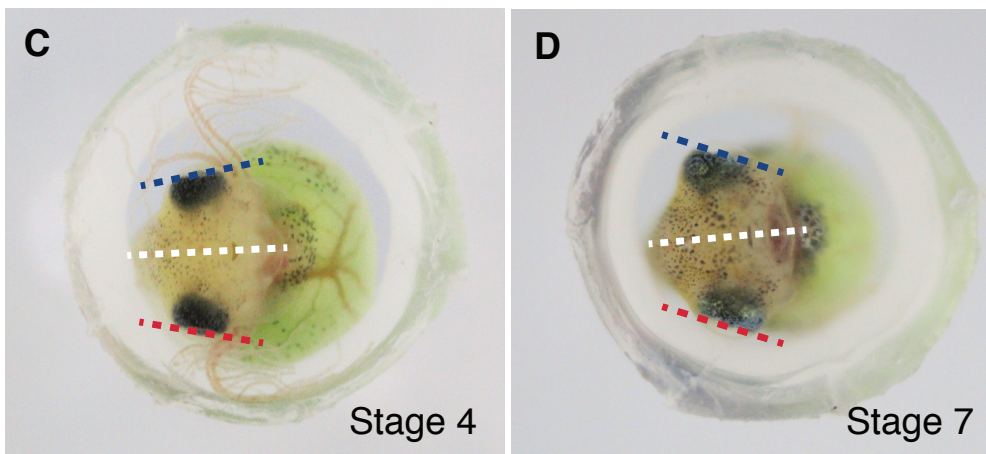

\section{Fig. 1. Example vestibulo-ocular reflex (VOR) curves for Agalychnis callidryas hatchlings.}

(A, B) Data points show eye angle (left in blue, right in red) plotted against body angle and lines are best fit curves for each eye. The peak-to-peak amplitude $\left(^{*}\right)$ of the curve measures the magnitude of VOR. (C, D) Frontal view of hatchlings tested, showing left (blue) and right (red) eye angles relative to the body axis (white). (A) Low VOR for a hatchling of age $3.6 \mathrm{~d}(\mathrm{C})$. (B) Clear VOR in a hatchling of age $4.5 \mathrm{~d}$ (D). 

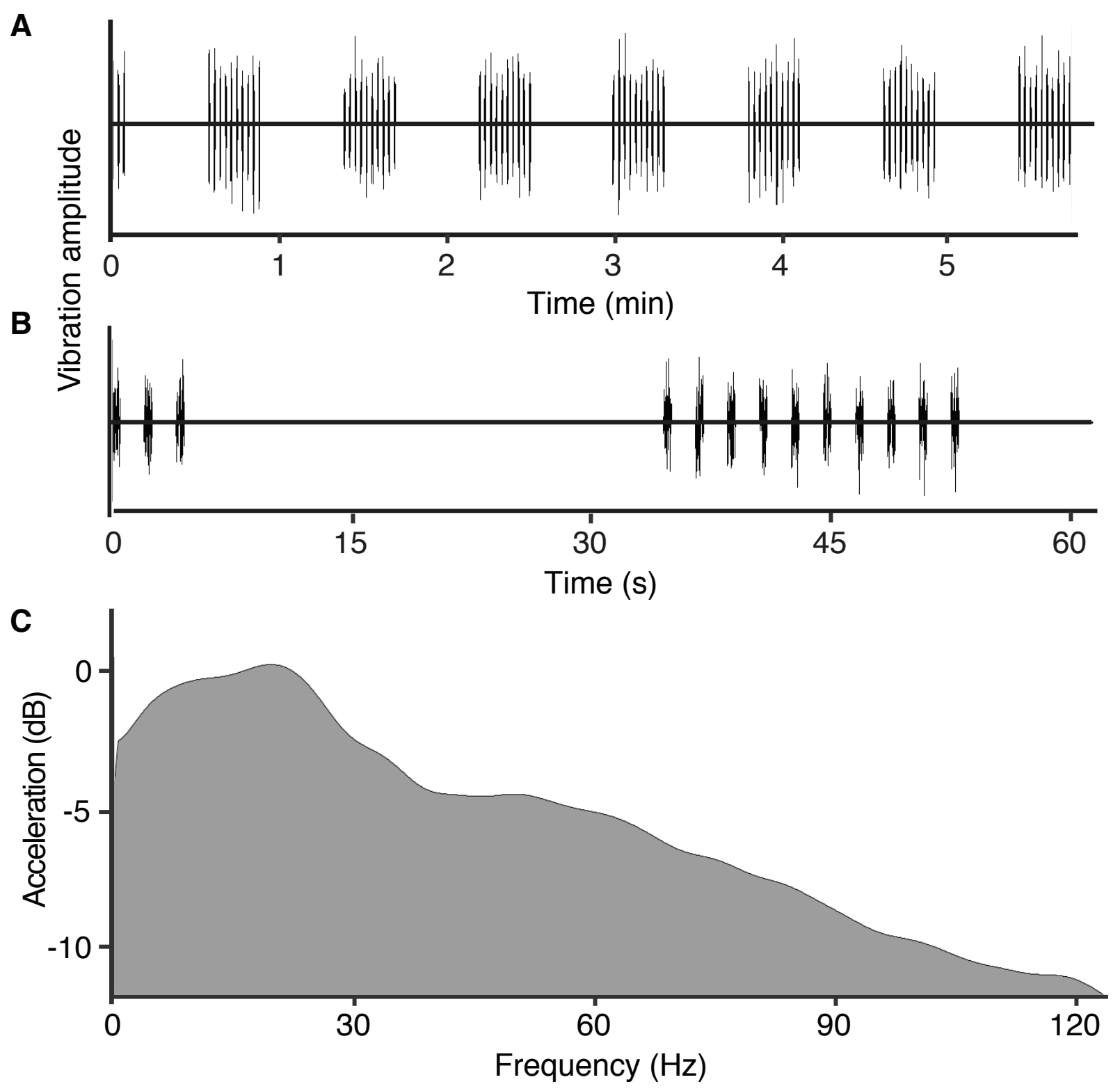

633 Fig. 2. Vibration playback stimulus. (A) The entire stimulus included 7 pulse groups divided 634 by $30 \mathrm{~s}$ gaps plus a 3-pulse primer before the first $30 \mathrm{~s}$ gap. (B) The first minute of the stimulus, 635 including the primer and one pulse group, comprised of ten $0.5 \mathrm{~s}$ pulses of vibration, separated 636 by $1.5 \mathrm{~s}$ intervals. (C) Frequency spectrum of acceleration, normalized from peak power. This 637 stimulus induces near 100\% hatching in 5-day-old clutches. 


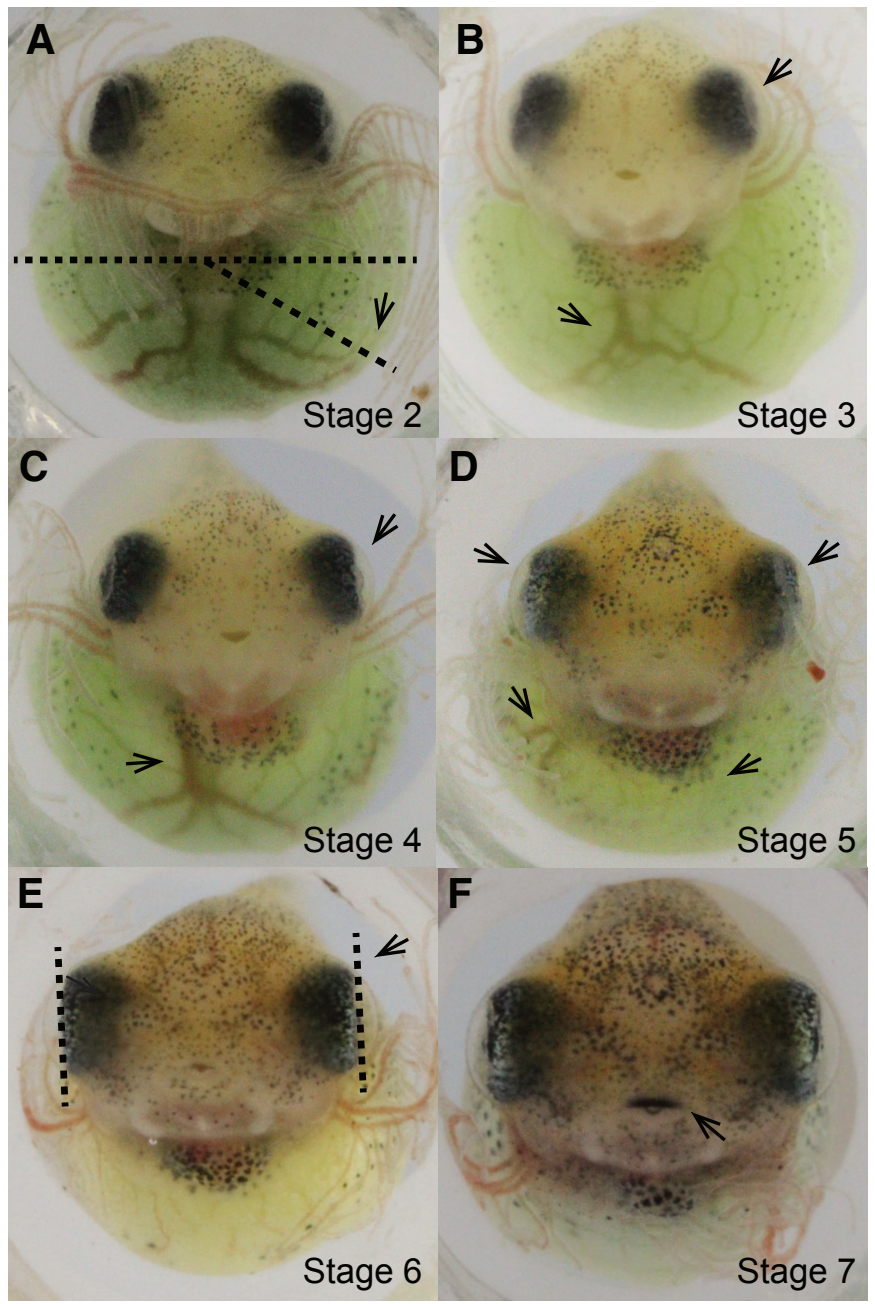

640 Fig. 3. Stages in the development of Agalychnis callidryas embryos through the onset of

641 hatching. Staging traits are adapted from Warkentin et al. (2017) and all visible in frontal view.

642 (A) Stage 2 - Melanophores extend at least halfway down sides over yolk. Two dominant veins

643 on yolk surface enter heart separately, fairly symmetrically under center of heart. (B) Stage 3 -

644 Two dominant veins on yolk surface join to enter heart as a single vessel. Cornea close to lens

645 and granular or slightly cloudy, partially obscures view of lens. (C) Stage 4 - Cornea clear and

646 well separated from lens; lens readily visible. Yolk vein enters medially below heart, within edge

647 of cement gland in direct frontal view, veins present in yolk posterior to heart. (D) Stage 5 -

648 Yolk vein enters heart dextrally, at or lateral to cement gland in direct frontal view, yolk

649 posterior to heart clear of veins. Eyes angled in dorsally. (E) Stage 6 - Eyes parallel in frontal

650 view, not angled in dorsally. Beaks unkeratinized. (F) Stage 7 - Edge of upper and/or lower

651 beaks keratinized, head narrower than yolk. 

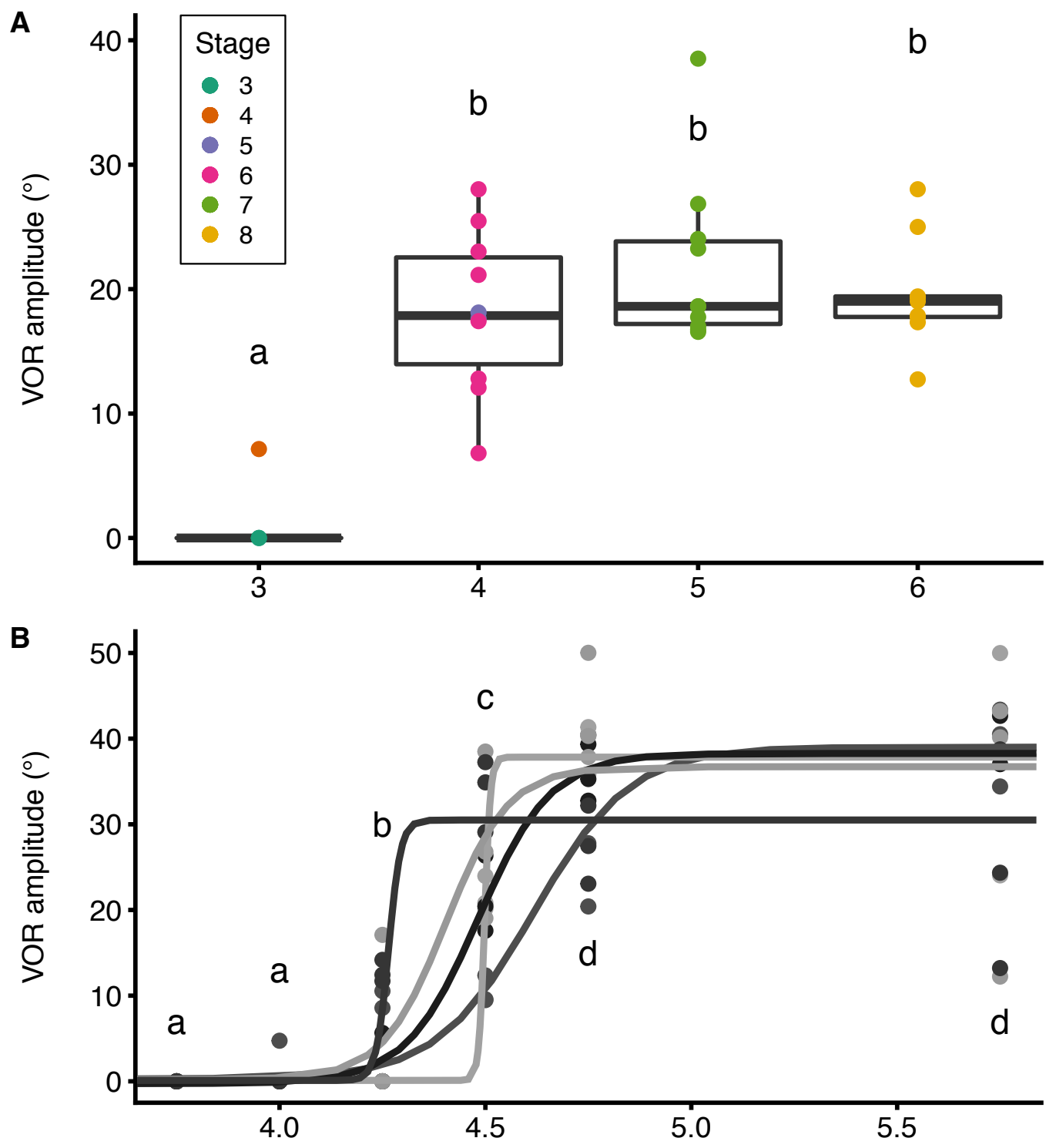

Developmental age (days)

653 Fig. 4. Ontogeny of the vestibulo-ocular reflex (VOR) across embryonic development of

654 Agalychnis callidryas. (A) Hatchings were tested at $24 \mathrm{~h}$ intervals from age 3-6 d (at

655 approximately $3 \mathrm{pm}$ ), using 7-10 non-sibling individuals per age (36 hatchlings tested from 14

656 different clutches). Individual hatchlings were tested for VOR immediately after hatching or

657 decapsulation and are color-coded by developmental stage. Box plots show medians,

658 interquartile range (IQR), and extent of data to $\pm 1.5 \times \mathrm{IQR}$. Different letters indicate significant

659 differences in VOR amplitudes between ages. (B) Individual hatchlings were tested from

660 developmental series of five clutches (shown in different shades of gray), with $\sim 3$ siblings per

661 test point (total $\mathrm{N}=88$ hatchlings; $\mathrm{N}=15,16,15,15,15,12$ per age). Lines show 4-parameter

662 logistic curve fit for each clutch. 


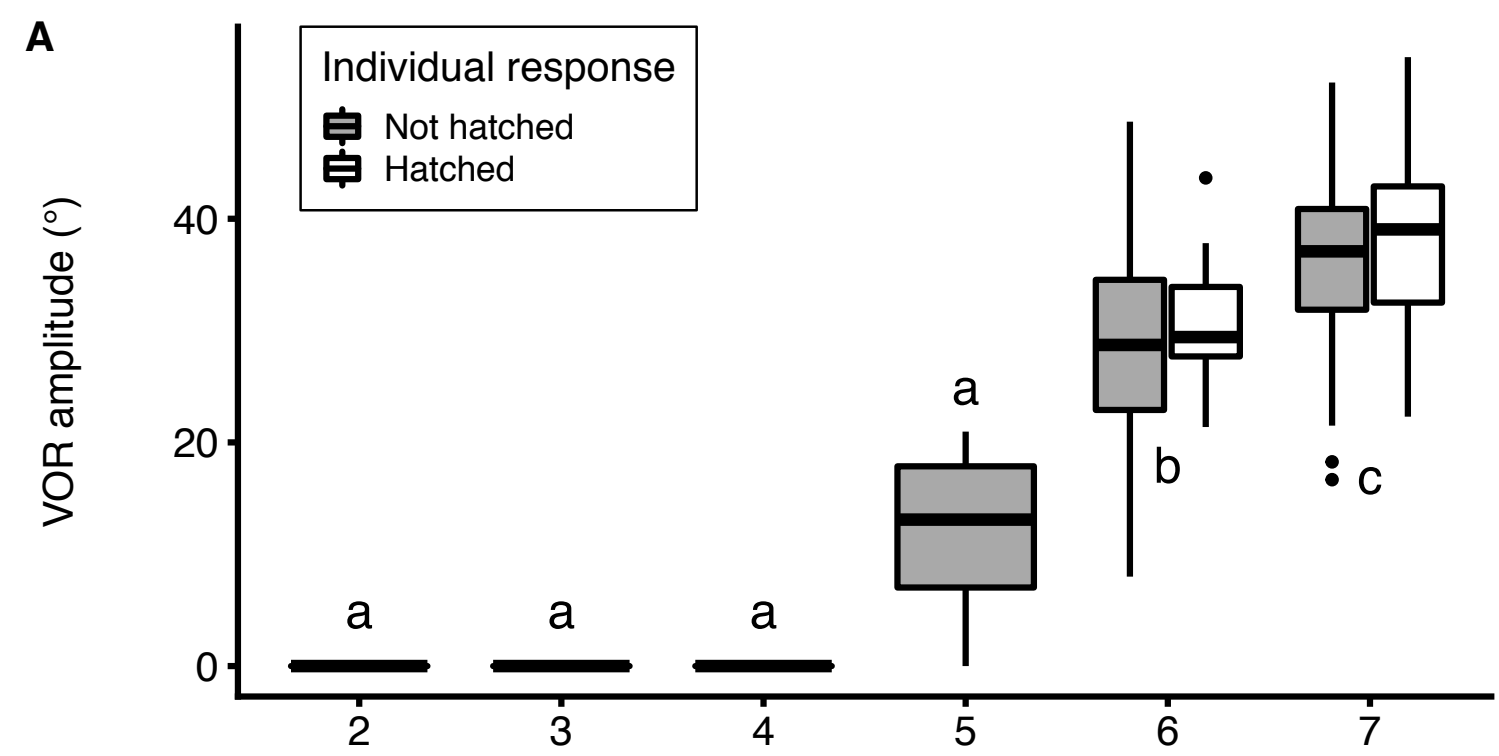

Developmental stage

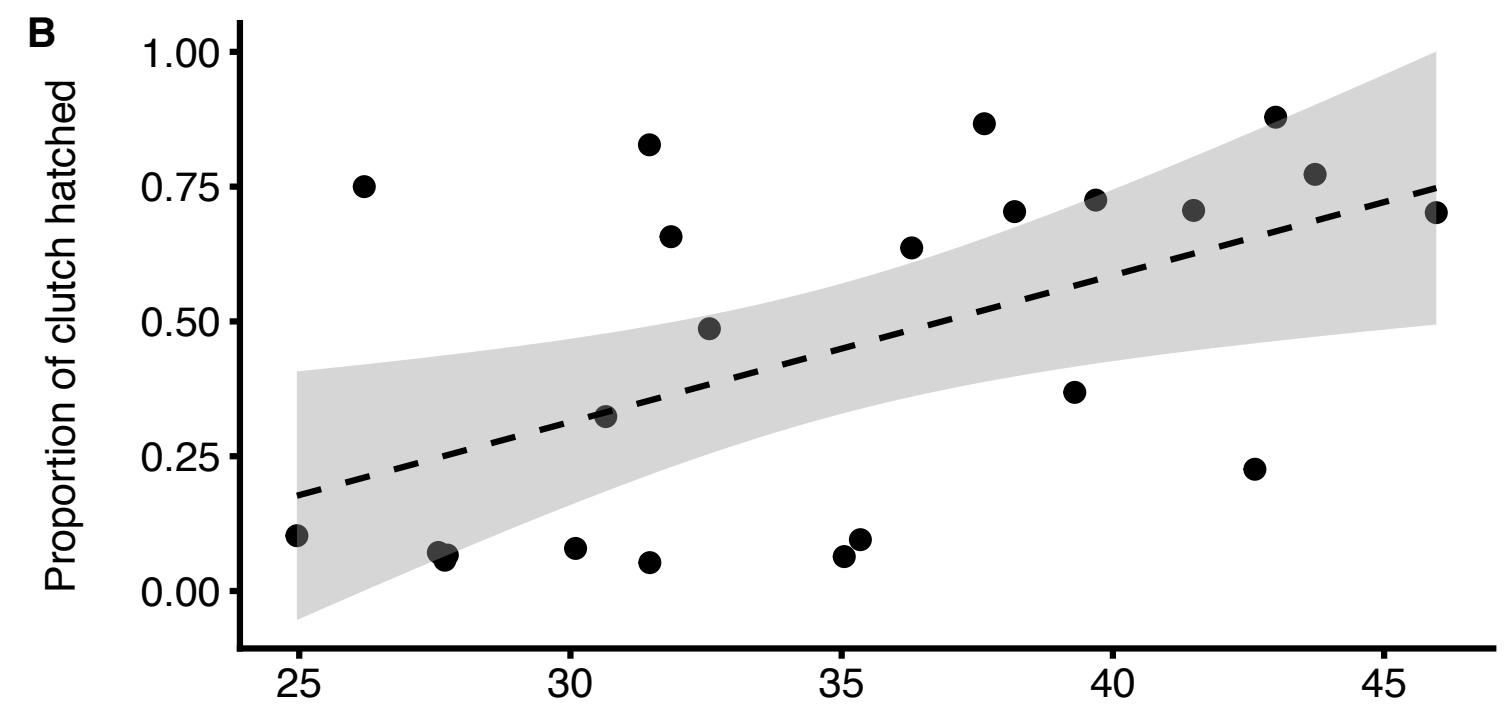

Clutch mean of VOR amplitude $\left({ }^{\circ}\right)$

663

664

665

666

667

668

669

670

671

672
Fig. 5. Relationships among vestibulo-ocular reflex (VOR) amplitude, development, and hatching response of Agalychnis callidryas embryos to vibration playbacks. (A) Filled and unfilled box plots indicate VOR of individuals $(\mathrm{N}=169)$ that did not hatch or hatched, respectively, in response to vibration playbacks to whole clutches across development. Box plots show medians, interquartile range (IQR) and extent of data to $\pm 1.5 \times \mathrm{IQR}$, and outliers as points. Different letters indicate significant differences between stages. (B) Total proportion hatched, for the 23 clutches from which at least one embryo hatched, plotted against clutch mean VOR amplitude measured from 169 hatchlings (4-6 individuals per clutch). Dashed line indicates the linear regression fit, shading indicates $95 \%$ confidence interval. 

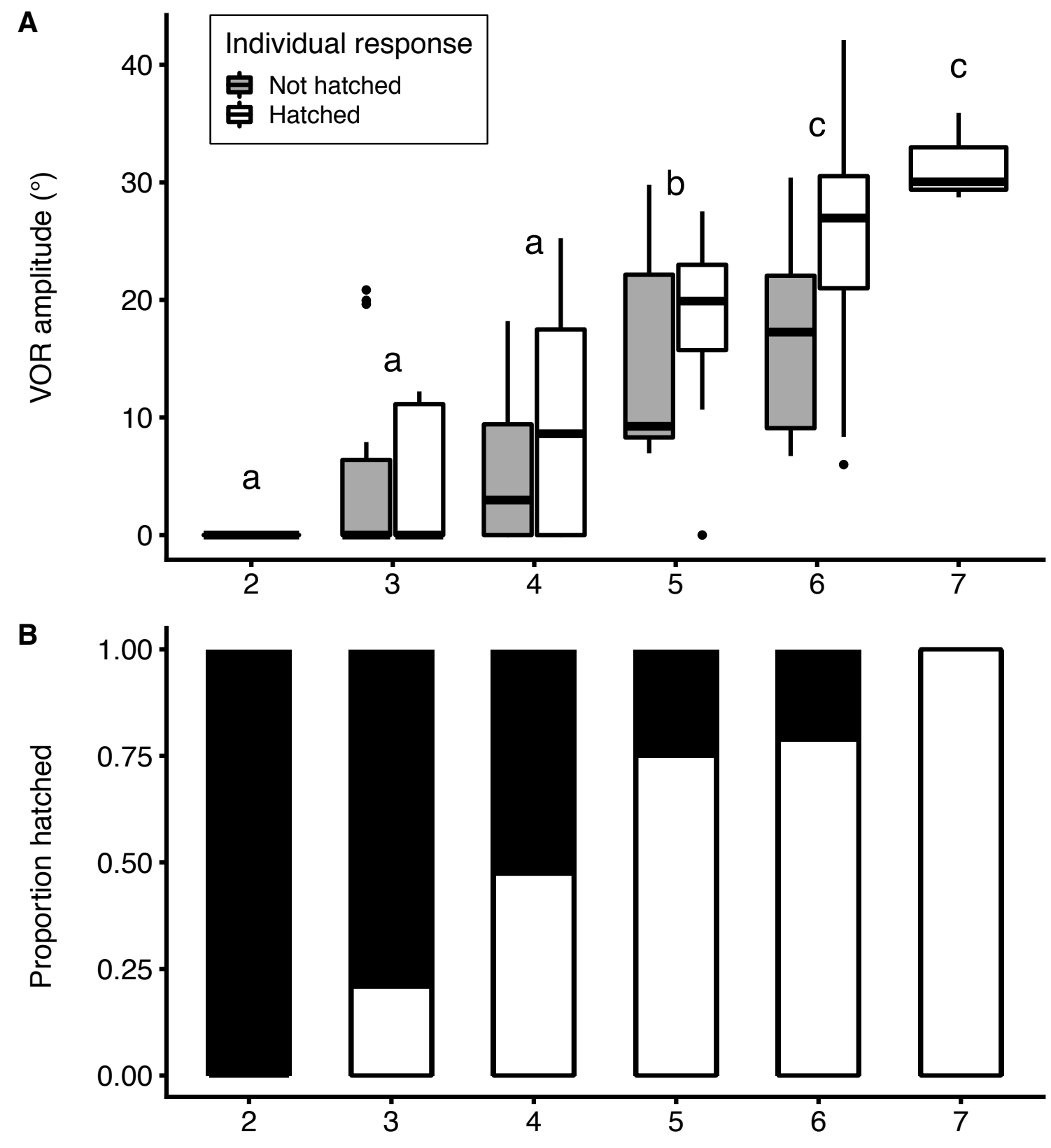

Developmental stage

675 Fig. 6. Ontogeny of vestibulo-ocular reflex (VOR) and hatching responses in Agalychnis

676 callidryas. (A) Ontogeny of VOR across stages, from developmental series of 11 egg clutches, 677 with two siblings tested per time point ( $\mathrm{N}=112$ individuals). Different letters indicate significant 678 differences between stages. Box plots show medians, interquartile range (IQR) and extent of data 679 to $\pm 1.5 \times \mathrm{IQR}$, and points show outliers. Filled and unfilled box plots indicate embryos that did 680 not hatch and hatched, respectively, in response to manual egg jiggling. (B) Ontogeny of 681 hatching response to manual jiggling of individual eggs. Proportion hatched (white boxes) is of 682 individual embryos tested per stage. 


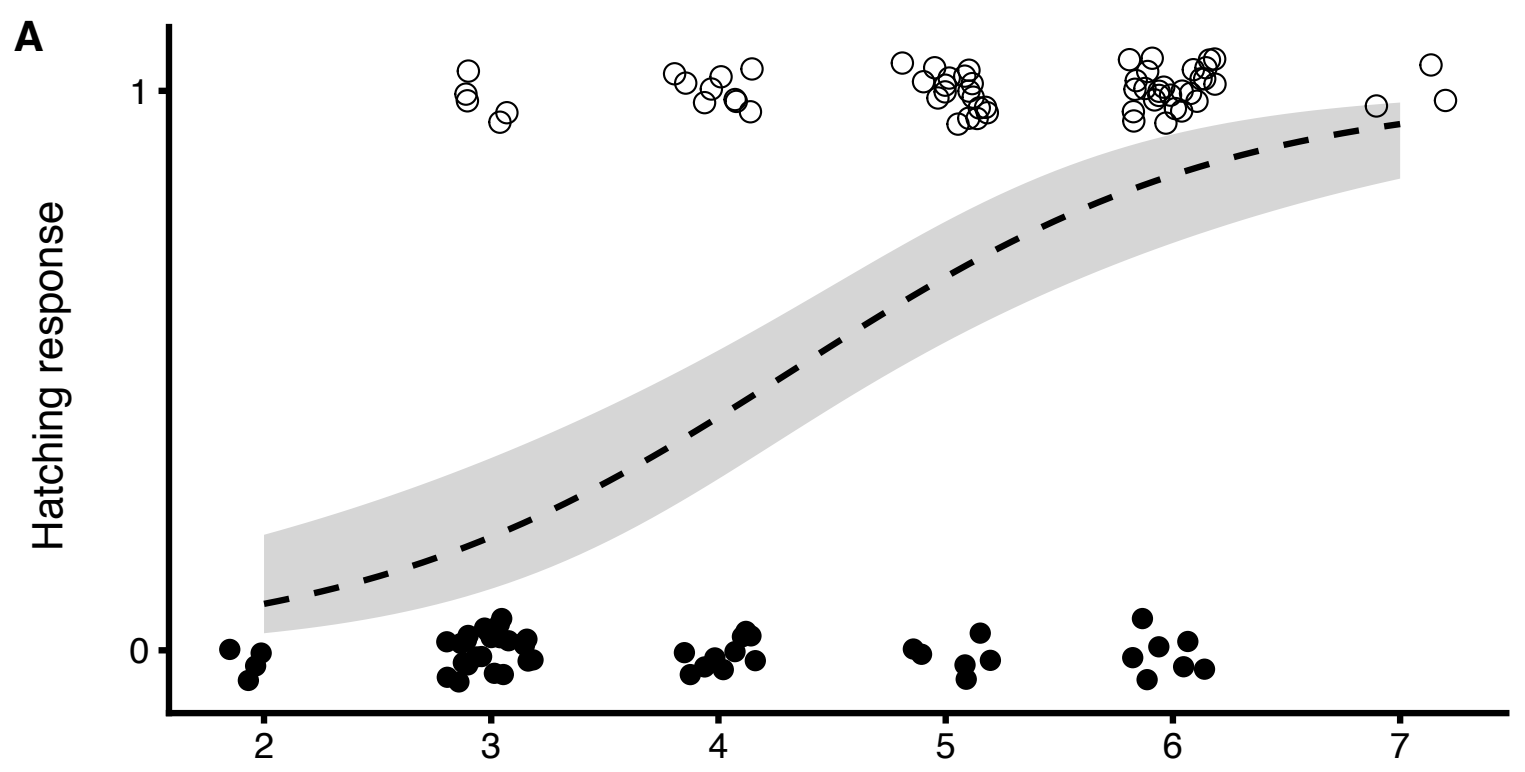

Developmental stage

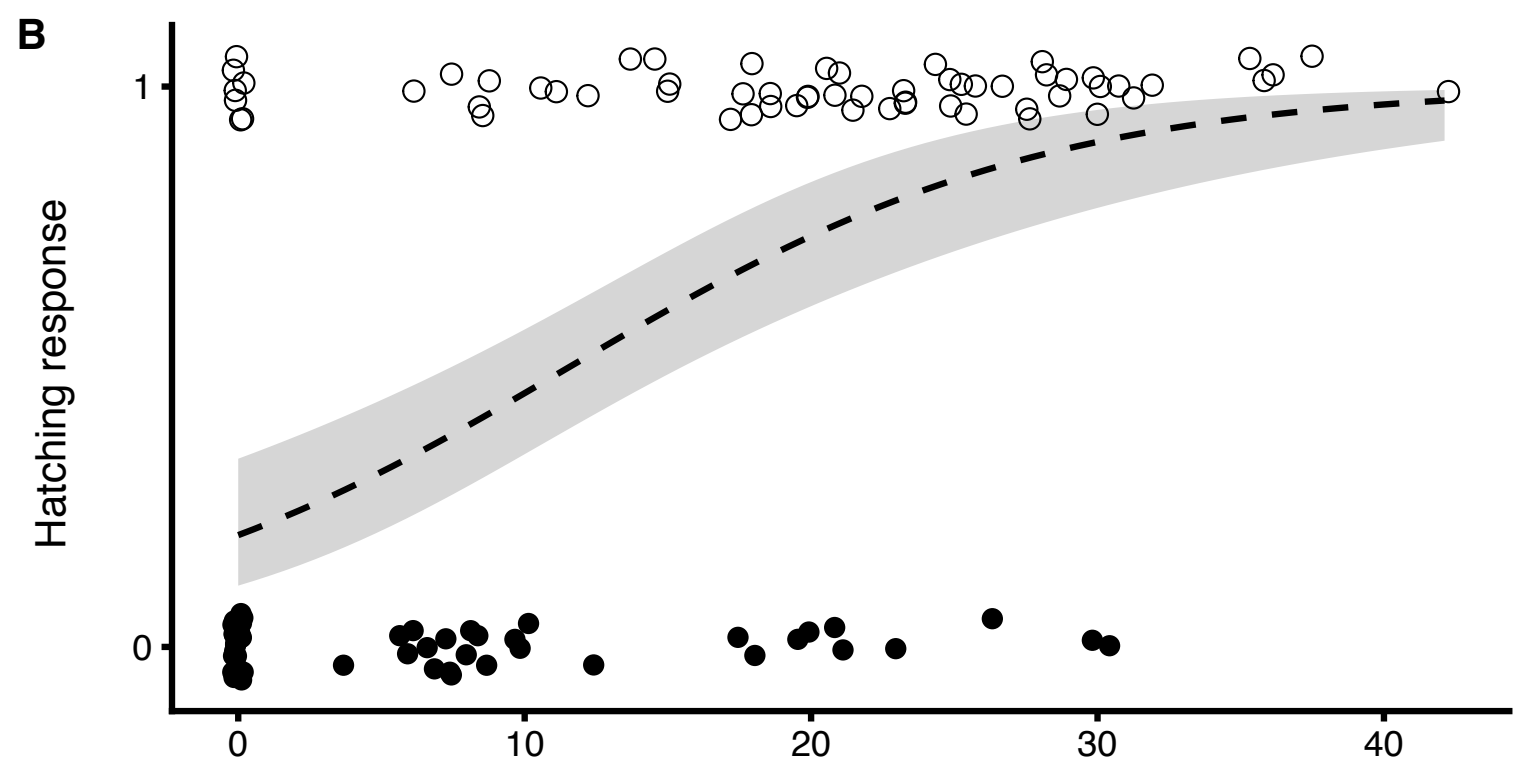
on hatching response of Agalychnis callidryas embryos to egg jiggling. Values of 0 (unhatched, filled circles) and 1 (hatched, open circles) are jittered vertically to show data points. Integer values (developmental stages) are jittered, while continuous individual measurements (VOR amplitude) are not. Dashed lines are predicted fits from binomial generalized linear mixed models; shading indicates the $95 \%$ confidence interval. 


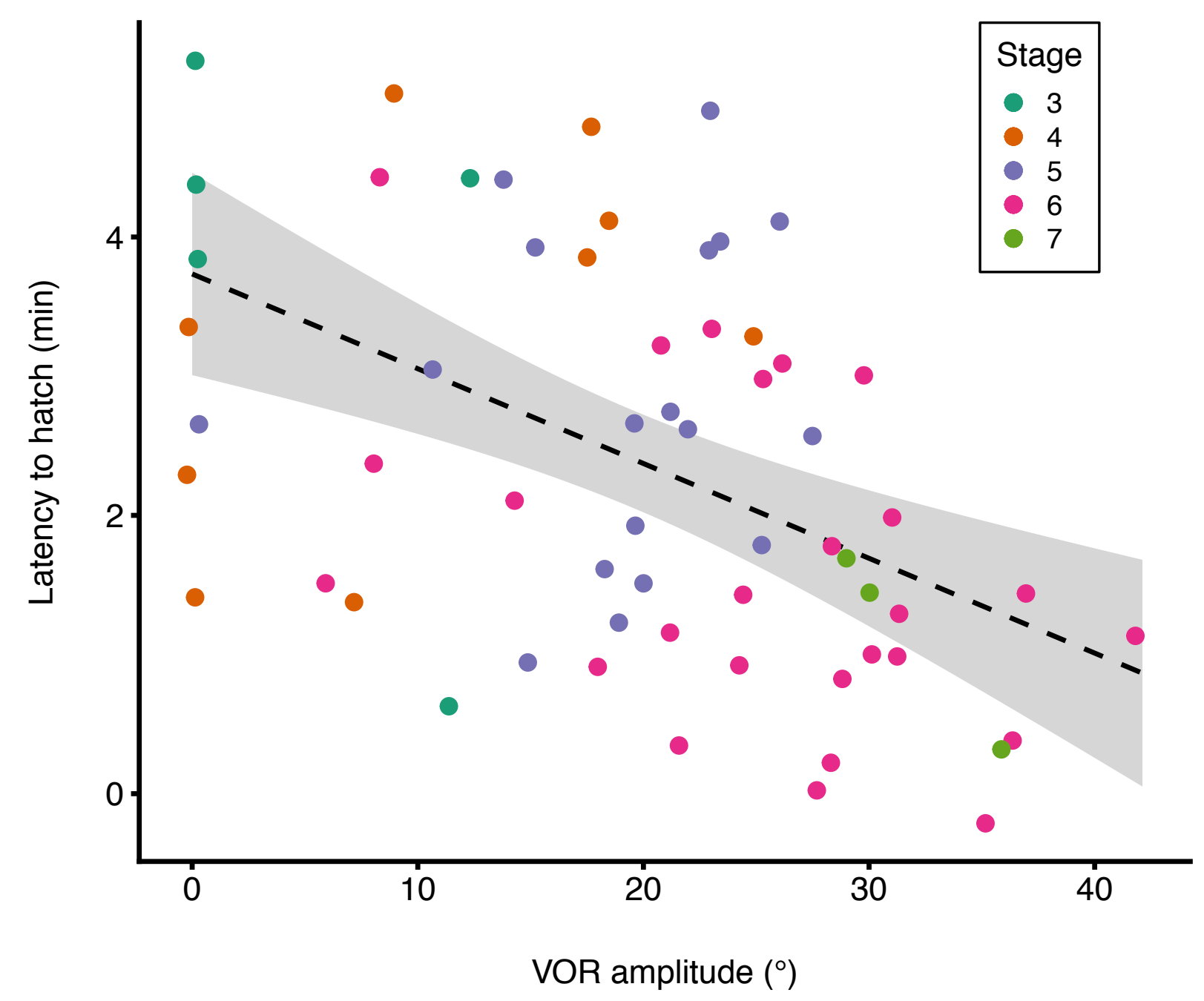

693 Fig. 8. Latency of Agalychnis callidryas embryos to hatch in response to egg jiggling, in 694 relation to vestibulo-ocular reflex (VOR) amplitude. Data are for $\mathrm{N}=61$ embryos tested 695 individually. Developmental stages are indicated by color. Dashed line indicates linear 696 regression fit; shading shows the 95\% confidence interval. 


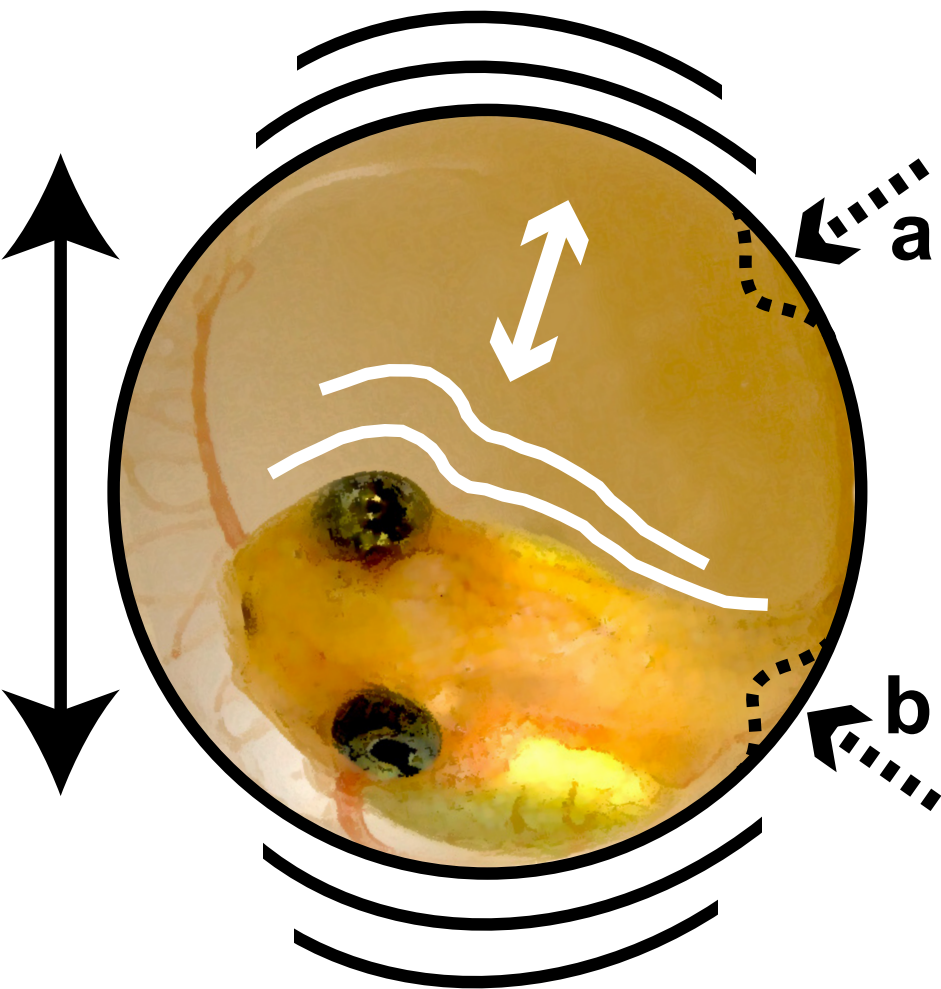

Fig. 9. Types of mechanosensory cues in egg-predator attacks. Attacked embryos may experience whole-egg motion (solid black), embryo motion within the perivitelline chamber (white), and direct contact with eggs (dashed) that may a. deform egg capsules or b. touch embryos through their capsule. 


\section{References}

Almanzar, A. and Warkentin, K. M. (2018). How development changes escape-hatching success in snake attacks: a video analysis of red-eyed treefrog embryo behavior and performance. Integr. Comp. Biol., P2-272.

Alsina, B. and Whitfield, T. T. (2017). Sculpting the labyrinth: morphogenesis of the developing inner ear. Semin. Cell Dev. Biol. 65, 47-59.

Becerra, M. and Anadon, R. (1993). Development of the inner-ear of the brown trout (Salmo trutta fario). 2. Cytodifferentiation and innervation of sensory cells. J. Morphol. 216, 241-257.

Bever, M. M. and Fekete, D. M. (2002). Atlas of the developing inner ear in zebrafish. Dev. Dyn. 223, 536-43.

Bever, M. M., Jean, Y. Y. and Fekete, D. M. (2003). Three-dimensional morphology of inner ear development in Xenopus laevis. Dev. Dyn. 227, 422-430.

Buckley, C. R., Michael, S. F. and Irschick, D. J. (2005). Early hatching decreases jumping performance in a direct-developing frog, Eleutherodactylus coqui. Funct. Ecol. 19, 67-72.

Caldwell, M. S., McDaniel, J. G. and Warkentin, K. M. (2009). Frequency information in the vibration-cued escape hatching of red-eyed treefrogs. J. Exp. Biol. 212, 566-575.

Caldwell, M. S., McDaniel, J. G. and Warkentin, K. M. (2010). Is it safe? Red-eyed treefrog embryos assessing predation risk use two features of rain vibrations to avoid false alarms. Anim. Behav. 79, 255-260.

Catton, W. T. (1976). Cutaneous mechanoreceptors. In Frog Neurobiology, a Handbook, eds. R. Llinás and W. Precht), pp. 629-642. Berlin: Springer-Verlag.

Cohen, B. (1974). The vestibulo-ocular reflex arc. In Vestibular System Part 1: Basic Mechanisms, (ed. H. H. Kornhuber), pp. 477-540. Berlin, Heidelberg: Springer Berlin Heidelberg.

Cohen, K. L., Piacentino, M. L. and Warkentin, K. M. (2019). Two types of hatching gland cells facilitate escape hatching at different developmental stages in red-eyed treefrogs, Agalychnis callidryas (Anura: Phyllomedusidae). Biol. J. Linn. Soc. 126, 751-767.

Cohen, K. L., Seid, M. A. and Warkentin, K. M. (2016). How embryos escape from danger: the mechanism of rapid, plastic hatching in red-eyed treefrogs. J. Exp. Biol. 219, 18751883.

Doody, J. S. (2011). Environmentally cued hatching in reptiles. Integr. Comp. Biol. 51, 49-61.

Doody, J. S. and Paull, P. (2013). Hitting the ground running: environmentally cued hatching in a lizard. Copeia 2013, 160-165. 
Doody, J. S., Stewart, B., Camacho, C. and Christian, K. (2012). Good vibrations? Sibling embryos expedite hatching in a turtle. Anim. Behav. 83, 645-651.

Endo, J., Takanashi, T., Mukai, H. and Numata, H. (2018). Egg-cracking vibration as a cue for stink bug siblings to synchronize hatching. Curr. Biol. 29, 143-148.

Fritzsch, B. (1996). How does the urodele ear develop? Int. J. Dev. Biol. 40, 763-771.

Fritzsch, B. (2003). Development of inner ear afferent connections: forming primary neurons and connecting them to the developing sensory epithelia. Brain Res. Bull. 60, 423-33.

Fritzsch, B., Barald, K. F. and Lomax, M. I. (1998). Early embryology of the vertebrate ear. In Development of the Auditory System, (ed. E. W. Rubel), pp. 80-145. New York: Springer-Verlag New York, Inc.

Fritzsch, B., Beisel, K. W., Jones, K., Farinas, I., Maklad, A., Lee, J. and Reichardt, L. F. (2002). Development and evolution of inner ear sensory epithelia and their innervation. $J$. Neurobiol. 53, 143-156.

Fromy, B., Sigaudo-Roussel, D. and L Saumet, J. (2008). Cutaneous neurovascular interaction involved in tactile sensation. Cardiovasc. Hematol. Agents. Med. Chem. 6, 337-42.

Gabriel, M., Frippiat, J. P., Frey, H. and Horn, E. R. (2012). The sensitivity of an immature vestibular system to altered gravity. J. Exp. Zool. A Ecol. Genet. Physiol. 317, 333-46.

Gilland, E. and Baker, R. (2005). Evolutionary patterns of cranial nerve efferent nuclei in vertebrates. Brain Behav. Evol. 66, 234-254.

Glover, J. C. (2003). The development of vestibulo-ocular circuitry in the chicken embryo. $J$. Physiol. Paris 97, 17-25.

Gomez-Mestre, I. and Warkentin, K. M. (2007). To hatch and hatch not: similar selective trade-offs but different responses to egg predators in two closely related, syntopic treefrogs. Oecologia 153, 197-206.

Gomez-Mestre, I., Wiens, J. J. and Warkentin, K. M. (2008). Evolution of adaptive plasticity: risk-sensitive hatching in neotropical leaf-breeding treefrogs. Ecol. Monogr. 78, 205-224.

Goyes Vallejos, J., Grafe, T. U. and Wells, K. D. (2018). Prolonged parental behaviour by males of Limnonectes palavanensis (Boulenger 1894), a frog with possible sex-role reversal. J. Nat. Hist. 52, 2473-2485.

Güell, B. A. and Warkentin, K. M. (2018). When and where to hatch? Red-eyed treefrog embryos use light cues in two contexts. PeerJ 6, e6018.

Haddon, C. and Lewis, J. (1996). Early ear development in the embryo of the zebrafish, Danio rerio. J. Comp. Neurol. 365, 113-28. 
Hill, P. S. M. (2008). Vibrational Communication in Animals. Cambridge, Massachusetts: Harvard University Press.

Hite, J. L., Hughey, M. C., Warkentin, K. M. and Vonesh, J. R. (2018). Cross-ecosystem effects of terrestrial predators link treefrogs, zooplankton, and aquatic primary production. Ecosphere 9, e02377.

Horn, E., Lang, H. G. and Rayer, B. (1986a). The development of the static vestibulo-ocular reflex in the southern clawed toad, Xenopus laevis. I. Intact animals. J. Comp. Physiol. A Sens. Neural Behav. Physiol. 159, 869-78.

Horn, E., Mack, R. and Lang, H. G. (1986b). The development of the static vestibulo-ocular reflex in the southern clawed toad, Xenopus laevis. II. Animals with acute vestibular lesions. J. Comp. Physiol. A Sens. Neural Behav. Physiol. 159, 879-85.

Horn, E. and Sebastian, C. (1996). A hypergravity related sensitive period during the development of the roll induced vestibuloocular reflex in an amphibian (Xenopus laevis). Neurosci. Lett. 216, 25-8.

Horn, E. R. (2006). Microgravity-induced modifications of the vestibuloocular reflex in Xenopus laevis tadpoles are related to development and the occurrence of tail lordosis. $J$. Exp. Biol. 209, 2847-58.

Horn, E. R., El-Yamany, N. A. and Gradl, D. (2013). The vestibuloocular reflex of tadpoles Xenopus laevis after knock-down of the isthmus-related transcription factor XTcf-4. $J$. Exp. Biol. 216, 733-741.

Horn, E. R. and Gabriel, M. (2011). Gravity-related critical periods in vestibular and tail development of Xenopus laevis. J. Exp. Zool. A Ecol. Genet. Physiol. 315, 505-11.

Hughey, M. C., Rogge, J. R., Thomas, K., McCoy, M. W. and Warkentin, K. M. (2015). Escape-hatching responses of individual treefrog embryos vary with threat level in wasp attacks: a mechanistic analysis. Behaviour 152, 1543-1568.

Hunt, C. C. and McIntyre, A. K. (1960). Properties of cutaneous touch receptors in cat. $J$. Physiol. 153, 88-98.

Jen, J. C. (2009). Bilateral vestibulopathy: clinical, diagnostic, and genetic considerations. Semin. Neurol. 29, 528-33.

Jenkins, B. A. and Lumpkin, E. A. (2017). Developing a sense of touch. Development 144, 4078.

Liang, J. K., Bok, J. and Wu, D. K. (2010). Distinct contributions from the hindbrain and mesenchyme to inner ear morphogenesis. Dev. Biol. 337, 324-34. 
Martin, K., Bailey, K., Moravek, C. and Carlson, K. (2011). Taking the plunge: California grunion embryos emerge rapidly with environmentally cued hatching. Integr. Comp. Biol. 51, 26-37.

Mogdans, J. and Bleckmann, H. (2012). Coping with flow: behavior, neurophysiology and modeling of the fish lateral line system. Biol. Cybern. 106, 627-642.

Mukai, H., Hironaka, M., Tojo, S. and Nomakuchi, S. (2014). Maternal vibration: an important cue for embryo hatching in a subsocial shield bug. Plos One 9.

Nieuwkoop, P. D. and Faber, J. (1956). Normal table of Xenopus laevis (Daudin); a systematical and chronological survey of the development from the fertilized egg till the end of metamorphosis. North-Holland, Amsterdam: North-Holland Pub. Co.

Nishide, Y. and Tanaka, S. (2016). Desert locust, Schistocerca gregaria, eggs hatch in synchrony in a mass but not when separated. Behav. Ecol. Sociobiol. 70, 1507-1515.

Oyarzun, F. X. and Strathmann, R. R. (2011). Plasticity of hatching and the duration of planktonic development in marine invertebrates. Integr. Comp. Biol. 51, 81-90.

Precht, W. (1976). Physiology of the peripheral and central vestibular systems. In Frog Neurobiology: A Handbook, pp. 481-512. Berlin, Heidelberg: Springer Berlin Heidelberg.

Pyburn, W. F. (1970). Breeding behavior of leaf-frogs Phyllomedusa callidryas and Phyllomedusa dacnicolor in Mexico. Copeia 1970, 209-218.

Quick, Q. A. and Serrano, E. E. (2005). Inner ear formation during the early larval development of Xenopus laevis. Dev. Dyn. 234, 791-801.

Rayer, B. and Horn, E. (1986). The development of the static vestibulo-ocular reflex in the southern clawed toad, Xenopus laevis. III. Chronic hemilabyrinthectomized tadpoles. J. Comp. Physiol. A Sens. Neural Behav. Physiol. 159, 887-895.

Ribot-Ciscar, E., Vedel, J. P. and Roll, J. P. (1989). Vibration sensitivity of slowly and rapidly adapting cutaneous mechanoreceptors in the human foot and leg. Neurosci. Lett. 104, 130-135.

Rogge, J. R. and Warkentin, K. M. (2008). External gills and adaptive embryo behavior facilitate synchronous development and hatching plasticity under respiratory constraint. J. Exp. Biol. 211, 3627-35.

Salica, M. J., Vonesh, J. R. and Warkentin, K. M. (2017). Egg clutch dehydration induces early hatching in red-eyed treefrogs, Agalychnis callidryas. PeerJ $\mathbf{5}$.

Schaefer, K. P. and Meyer, D. L. (1974). Compensation of vestibular lesions. In Vestibular System Part 2: Psychophysics, Applied Aspects and General Interpretations, (ed. H. H. Kornhuber), pp. 463-490. Berlin, Heidelberg: Springer Berlin Heidelberg. 
Schneider, C. A., Rasband, W. S. and Eliceiri, K. W. (2012). NIH Image to ImageJ: 25 years of image analysis. Nat. Meth. 9, 671-5.

Sebastian, C., Esseling, K. and Horn, E. (2001). Altered gravitational forces affect the development of the static vestibuloocular reflex in fish (Oreochromis mossambicus). $J$. Neurobiol. 46, 59-72.

Sebastian, C. and Horn, E. (1999). Light-dependent suppression of the vestibulo-ocular reflex during development. Neuroreport 10, 171-6.

Sih, A. and Moore, R. D. (1993). Delayed hatching of salamander eggs in response to enhanced larval predation risk. Am. Nat. 142, 947-960.

Speer-Blank, T. M. and Martin, K. L. M. (2004). Hatching events in the California grunion, Leuresthes tenuis. Copeia 2004, 21-27.

Spray, D. C. (1976). Pain and temperature receptors of anurans. In Frog Neurobiology, a Handbook, eds. R. Llinás and W. Precht), pp. 607-628. New York: Springer-Verlag.

Stone, L. S. (1933). The development of lateral-line sense organs in amphibians observed in living and vital-stained preparations. J. Comp. Neurol. 57, 507-540.

Straka, H. (2010). Ontogenetic rules and constraints of vestibulo-ocular reflex development. Curr. Opin. Neurobiol. 20, 689-695.

Strelioff, D. and Honrubia, V. (1978). Neural transduction in Xenopus laevis lateral line system. $J$. Neurophysiol. 41, 432-44.

Tanaka, S., Harano, K. I., Nishide, Y. and Sugahara, R. (2016). The mechanism controlling phenotypic plasticity of body color in the desert locust: some recent progress. Curr. Opin. Insect Sci. 17, 10-15.

Thomas, E. D., Cruz, I. A., Hailey, D. W. and Raible, D. W. (2015). There and back again: development and regeneration of the zebrafish lateral line system. Wiley. Interdiscip. Rev. Dev. Biol. 4, 1-16.

Touchon, J. C., Urbina, J. and Warkentin, K. M. (2011). Habitat-specific constraints on induced hatching in a treefrog with reproductive mode plasticity. Behav. Ecol. 22, 169175.

Warkentin, K. M. (1995). Adaptive plasticity in hatching age: a response to predation risk trade-offs. Proc. Natl. Acad. Sci. U.S.A. 92, 3507-3510.

Warkentin, K. M. (1999). Effects of hatching age on development and hatchling morphology in the red-eyed treefrog, Agalychnis callidryas. Biol. J. Linn. Soc. 68, 443-470.

Warkentin, K. M. (2000). Wasp predation and wasp-induced hatching of red-eyed treefrog eggs. Anim. Behav. 60, 503-510. 
Warkentin, K. M. (2002). Hatching timing, oxygen availability, and external gill regression in the tree frog, Agalychnis callidryas. Physiol. Biochem. Zool. 75, 155-164.

Warkentin, K. M. (2005). How do embryos assess risk? Vibrational cues in predator-induced hatching of red-eyed treefrogs. Anim. Behav. 70, 59-71.

Warkentin, K. M. (2011a). Environmentally cued hatching across taxa: embryos respond to risk and opportunity. Integr. Comp. Biol. 51, 14-25.

Warkentin, K. M. (2011b). Plasticity of hatching in amphibians: evolution, trade-offs, cues and mechanisms. Integr. Comp. Biol. 51, 111-127.

Warkentin, K. M., Buckley, C. R. and Metcalf, K. A. (2006a). Development of red-eyed treefrog eggs affects efficiency and choices of egg-foraging wasps. Anim. Behav. 71, 417425 .

Warkentin, K. M., Caldwell, M. S. and McDaniel, J. G. (2006b). Temporal pattern cues in vibrational risk assessment by embryos of the red-eyed treefrog, Agalychnis callidryas. $J$. Exp. Biol. 209, 1376-1384.

Warkentin, K. M., Cuccaro Diaz, J., Güell, B. A., Jung, J., Kim, S. J. and Cohen, K. L. (2017). Developmental onset of escape-hatching responses in red-eyed treefrogs depends on cue type. Anim. Behav. 129, 103-112.

Warkentin, K. M., Gomez-Mestre, I. and McDaniel, J. G. (2005). Development, surface exposure, and embryo behavior affect oxygen levels in eggs of the red-eyed treefrog, Agalychnis callidryas. Physiol. Biochem. Zool. 78, 956-66.

Warkentin, K. M., Jung, J., Rueda Solano, L. A. and McDaniel, J. G. (2019). Ontogeny of escape-hatching decisions: vibrational cue use changes as predicted from costs of sampling and false alarms. Behav. Ecol. Sociobiol. 73, 51.

Weeg, M. S. and Bass, A. H. (2002). Frequency response properties of lateral line superficial neuromasts in a vocal fish, with evidence for acoustic sensitivity. J. Neurophysiol. 88, $1252-62$.

Weston, J. A. (1970). The migration and differentiation of neural crest cells. In Advances in Morphogenesis, vol. 8 eds. M. Abercrombie J. Brachet and T. J. King), pp. 41-114: Elsevier.

Whittington, I. D. and Kearn, G. C. (1988). Rapid hatching of mechanically-disturbed eggs of the monogenean gill parasite Diclidophora luscae, with observations on sedimentation of egg bundles. Int. J. Parasitol. 18, 847-852.

Willink, B., Palmer, M. S., Landberg, T., Vonesh, J. R. and Warkentin, K. M. (2014). Environmental context shapes immediate and cumulative costs of risk-induced early hatching. Evol. Ecol. 28, 103-116. 